to appear in: L. Albertazzi, G. van Tonder \& D. Vishwanath (eds.). Perception beyond Inference. The Information Content of Visual Processes. (pp. 159-200). Cambridge, Mass: MIT Press. .

\title{
The perception of material qualities and the internal semantics of the perceptual system
}

\author{
Rainer Mausfeld
}

\section{Introduction}

The perception of material qualities poses a pre-eminent challenge for perception theory and provides an instructive study case for probing the soundness and explanatory force of theoretical frameworks for perception. Phenomena pertaining to the perception of material appearances are particularly suited to expose the explanatory gap between the information available in the sensory input and the meaningful categories that characterise the output of the perceptual system. More than seemingly elementary attributes, such as shape or colour, material appearances impart objects their meaningful properties of, say, being soft, wet, malleable, silky, juicy, edible, or deformable. We are obviously endowed with a specific perceptual capacity by which we can visually attain aspects of perceptual objects that pertain to their 'hidden' dispositional powers and propensities. Due to this capacity, we can not only identify specific kinds of objects and stuff, but we can also visually grasp an abundant variety of properties of objects that go far beyond purely visual attributes. This capacity is part of our more general perceptual capacity for making causal assignments and for embedding all of our experiences into various kinds of internal causal analyses. The specific kind of dispositional properties and causal ascriptions that are perceptually accessible from the sensory data is subordinated to the type of perceptual object that is activated by the sensory data. In the case of 'surfaces' - understood as perceptual objects, not as physical ones - these dispositional properties pertain to material qualities, and the causal ascriptions to, e.g., how surfaces will appear under changes in their orientation and location, which haptic experiences will be elicited by them, and how they will behave under various kinds of interactions, both with an agent and with other objects.

Although the capacity of visually attaining dispositional properties of objects constitutes one of the most remarkable functional achievements of our perceptual system, this capacity has received scant attention in traditional perceptual psychology. Apart from a few exceptions, investigations of the principles underlying the perception of material qualities have been largely neglected in favour of investigations of seemingly simpler attributes, such as shape or colour. This is a peculiar situation because investigations into the visual perception of material qualities of, say 'surfaces', promises to be a particularly rewarding field for the attempt to identify more abstract principles of perception. The general principles that are brought to bear by this achievement pertain to the perceptual system's capacity to attain the biologically important dispositional properties of its perceptual objects by a 'reading-through', as it were, of 
the available sensory image. A theoretical framework that is general enough to deal in an explanatorily satisfactory way with material qualities of 'surfaces', such as shiny, wet, deformable or walkable, is, or so I shall argue, also suited to appropriately deal with corresponding achievements with respect to other types of perceptual objects. As regards to perceptual objects of the type 'living animal', for example, these achievements comprise the capacity to perceptually attain 'hidden powers' and 'essences' of those types of objects; as regards to perceptual objects of the type 'mykind', they comprise the capacity to read through the object's surfaces in order to perceptually attain what we can refer to as 'mental states of others'. This can be strikingly illustrated by the demonstrations provided by Heider \& Simmel (1944), which show that certain kinds of motions of simple geometrical objects suffice for eliciting a perceptual ascription of complex internal attributes pertaining to mental states of 'mykind'. (cf. Scholl \& Tremoulet, 2000). Available evidence suggests, or so I will claim, that there is no difference of principle, with respect to the fundamental mode of operation of the perceptual system, between the perception of material qualities and the perception of, say, mental states of others. Although corresponding theoretical ideas have been widely expressed and elaborated since the $17^{\text {th }}$ century, current orthodoxy has pursued a different path of thinking. According to regnant conceptions in perceptual psychology, the perception of material qualities of surfaces is regarded as different in principle from the perception of dispositions of, say, a living object, or the perception of mental states of mykind. Although the first kind of dispositions are presumed to be attainable more or less 'directly' from complex spatio-temporal properties of the sensory input, the perception of the latter dispositions is presumed to rest on alleged 'higher-order' or 'cognitive' processes by which these dispositions were inferred from the sensory data. The fundamental flaws underlying corresponding conceptions have been disclosed again and again, notably by the Gestaltists. Although compelling empirical and theoretical evidence has been accumulated exposing the utter inadequacy of corresponding conceptions, they still dominate the field. Such a situation is, though, not unusual in the history of the natural sciences. It chiefly emerges when there is a clash between theoretical concepts that are strongly suggested by the available experimental evidence and deeply engrained common-sense notions. In fact, the propensity to cling to conceptions whose inadequacy, in light of the available evidence, stands to reason can itself be used as an expedient clue for the influence of tacit common-sense intuitions. With respect to physics, it is well-known that its entire history can be read as a continuous quarrel with common-sense intuitions. What holds for physics, even stronger holds for perception theory. We are held captive by the way perception appears to us.

We are strongly convinced that perceiving keeps us in more or less direct contact with the world and that what we perceive by and large is the world as it really is. Our ordinary conception about perception discounts, on the basis of an important functional achievement of the brain, all the processes that occur between the distal causes and the percept, and thus considers perception an entirely conspicuous process. Of course, we are willing to accept all sorts of sophistications and, under unusual circumstances, exceptions of this account, but otherwise regard it as a kind of truism. We thus have great difficulties in being aware that almost all of our ordinary intuitions about perception become misconceptions when transposed to the field of perception theory. Our brain is not equipped with mechanisms by which we can observe its 
own machinery, and the most fundamental principles underlying perception are, consequently, not transparent to us. Because of this, our ordinary intuitions about perception are an inapt guide for the endeavour to achieve, within the framework of the natural sciences, a theoretical understanding of the principles on which our perceptual achievements are based. Rather, a theoretical understanding of these principles can only be achieved by standard methodological principles of the natural sciences, which have proved highly fruitful in developing appropriate explanatory frameworks, often at the expense of sacrificing deeply valued common-sense intuitions. In speaking of ordinary or common-sense conceptions about perception, I will, in the present context, understand the term in the broadest possible way, namely as the diversity modes in which we conceive of perceptual phenomena and the process of perception itself in all contexts other than that of the natural sciences. (This usage comprises not only those concepts and ways of world-making that underlie, as part of our biological endowment, our ordinary discourse about the world and our acts of perceiving but also derived concepts and notions pertaining to perceptual issues that have been developed for other purposes than those of the natural sciences, whether technological, philosophical or of any other kind.)

Since the historic origin of rational enquiries, common-sense intuitions about perception have constituted the severest and almost insurmountable obstacle for the development of theoretical insights into the abstract principles on which our perceptual achievements are based. Enquiries into the nature of perception naturally have much greater difficulties than physics had to dispense with ordinary intuitions and common-sense classifications of phenomena and to instead follow lines of theorising that are traced out by the development of successful explanatory accounts. During recent decades, a theoretical convergence of different disciplines such as ethology, research with babies, and perceptual psychology has made more visible the contours of a general theoretical framework for a deeper understanding of perception. These developments, however, have not yet gained wider acceptance because they are grossly at variance with our common-sense conceptions about perception. Yet, this sacrifice of common-sense intuitions about perception will repay itself amply by the gain in explanatory width and depth of the theoretical insights into the abstract principles on which the achievements of the perceptual system are based.

In this chapter, I will attempt to flesh out the abstract theoretical framework that is currently (re-)emerging in the course of a theoretical convergence of several disciplines. In the first section, I shall formulate what appears to me to be the fundamental problem of perception theory, namely the generation, by the perceptual system, of meaningful categories from physico-geometric energy patterns. In the second section, I shall briefly characterise basic intuitions and assumptions underlying what can be regarded as the current Standard Model of Perceptual Psychology and point out why this model is profoundly inadequate for dealing with the fundamental problem of perception theory. In the third section, I shall suggest a level of analysis that promises to be fruitful for dealing, in conformity with established procedures of the natural sciences, with the problem of perceptual 'meaning' and the problem of what constitutes a 'perceptual object'. In the fourth section, I shall adumbrate the outlines of the theoretical perspective on basic principles of the perceptual system, as it appears to be emerging from the 
convergence of different disciplines. This approach, which centres on the notions of complex data types and conceptual forms, draws an entirely different theoretical picture of the role of the sensory input than traditional accounts. In the final section, I shall return to the issue of material qualities and discuss, within the general theoretical framework outlined, some observations and results on the perception of certain material properties, namely lustrous and glassy appearances.

\section{The Fundamental Problem of Perception Theory}

Perception theory, as I will understand it here, aims at a theoretical understanding of the basic principles on which our perceptual achievements are based; it proceeds on the assumption that these achievements are brought forth by a specific type of biological system, the perceptual system (which presumably is made up of several subsystems). Although perception theory acknowledges that the full range of our perceptual phenomena results from complex interactions of the perceptual system with other systems of the brain, it assumes that the proprietary mode of functioning of the perceptual system can be abstractly characterised by a set of fundamental principles. Hence it assumes that a broad range of core perceptual phenomena can be understood from a rather small set of theoretical principles. Perception theory thus proceeds on the standard methodological path of the natural sciences: It assumes that the apparent complexity of a certain set of phenomena derives from simple, and hence more abstract, principles that reflect the fundamental features of the idealised domain of nature under scrutiny. More specifically, perception theory assumes that the perceptual system, like other systems of the brain, can be regarded as a computational system (understood here in a rather loose sense), i.e. as a system that performs specific types of abstract operations on its input and its internal data structures, and delivers certain types of outputs to other systems. It furthermore employs the idealisation that the perceptual system can, to some interesting extent, be investigated independently of other computational systems of the brain, such as systems comprising motoric or linguistic representations, systems dealing with imaginations, or general interpretative or inferential systems. Because of these assumptions, perception theory will inevitably abstract away from a wealth of phenomena that appear to us striking from the perspective of our ordinary intuitions. As in other domains of the natural sciences, perception theory is willing to trade off descriptive richness for a gain in theoretical understanding, of sufficient widths and depth, of the fundamental principles on which the mode of operation of the perceptual system rests.

If we attempt to formulate what seems to be the fundamental problem of perception theory, we thus have to be aware that we are aiming at an explanatory account of the core principles of a specific system, namely the perceptual system (rather than providing an account for, say, achievements that are crucially based on the interactions of a variety of different subsystems or even intrinsically pertain to the entire organism). As a computational system, the perceptual system owns, on the one hand, a type of interface that connects it with physical states external to the organism, and, on the other hand, several types of internal interfaces by which it con- 
nects with the motoric system and higher imaginative, interpretative or linguistic systems. The standard input to the perceptual system can be characterised by a physical spatio-temporal energy pattern (chemical or interoceptive types of inputs can be subsumed under this abstract characterisation). In order to characterise its output, we first need to specify to which type of internal interface we want to refer. Naturally, all outputs of the perceptual system have to be organised in terms of data formats that can be read by the respective subsequent systems. Among these subsequent systems are ones that make some part of the output of the perceptual system phenomenologically accessible to us. The phenomenal percept and its internal organisation is not only the most salient aspect of perception for us, it also mirrors a crucial property of the output of the perceptual system, viz. its organisation in terms of meaningful categories. This property of an organisation in terms of internally meaningful categories applies to all types of outputs that the perceptual system provides at its interfaces to subsequent systems. By way of example, we can formulate the Fundamental Problem of Perception Theory for those types of outputs that are also phenomenally expressed.

As an illustration, take an apparently simple visual scene, such as the one depicted in figure 1. Although the visual input can be described as a geometrical luminance pattern on the retina, the output of the perceptual system, as mirrored in our phenomenal percept, is exceedingly rich and goes far beyond the input.

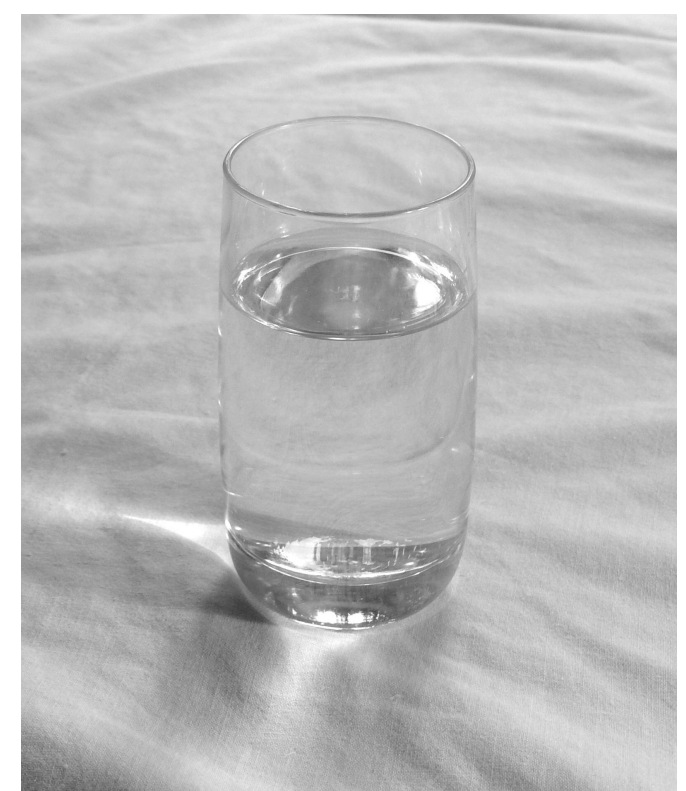

Figure 1: Illustration of material qualities

Perception theory thus has to deal with the question: What internal principles do we need to ascribe to the system under scrutiny in order to account for the finding that physical spatiotemporal energy patterns can yield an organisation of the output in terms of all the meaningful categories into which we segment our perceptual world. In the present examples, these categories refer, e.g., to ,container' with the attributes 'breakable' or 'transparent', and to ,water', with the attribute 'liquid' or 'drinkable'. 


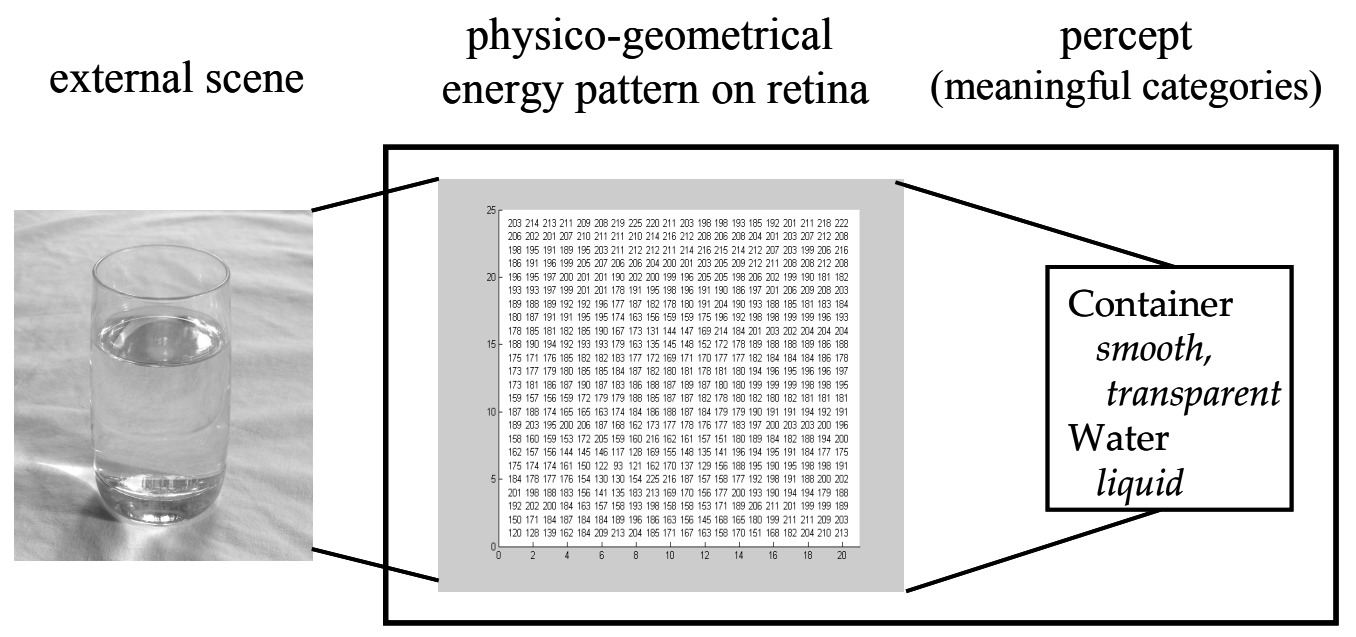

Figure 2: Illustration of the explanatory gap in perception theory

Figure 2 is a first stab at graphically depicting this problem. However, this way of describing the perceptually relevant entities of the external world is dictated by our common-sense conception of perception. What is depicted here as being the input is, at the same time, also the semantically organised percept, and thus the output of the system. Furthermore, our commonsense intuitions abet us to object to the type of abstract description taken in figure 2 to be the output. Rather, we would prefer to describe the output more directly in terms of phenomenal categories. Thus, common-sense intuitions lead us to a formulation by which input and output are basically identified, as allegorised in figure 3. This illustration caricatures, not entirely unduly, the naïve realism that is deeply built-in into our common-sense conception of perception: At the core of our quotidian conception of perception is the belief that the external world can be basically characterised by the way it appears to us, and that therefore the categories of our percepts are nothing but categories of the external world. In fact, the predisposition to take perceptual concepts for 'things in the real world' is the distinguishing mark of all of our mental activity. Kant thought of it as a "transcendental illusion". The transcendental illusion is the propensity to "take a subjective necessity of a connection of our concepts... for an objective necessity in the determination of things in themselves" (Critique of pure reason, A297/B354). Due to this propensity, whose influence cannot be remedied by intellectual insight into it, we inevitably tend to mistake our own mental categories to hold 'objectively' (cf. Grier, 2001). Scientific enquiry has shown how deep in fact the abyss is between what we take to be the 'external mind-independent world' and the world as conceptualised in human perception. Even in cases, where our linguistic vocabulary (as also employed in physical discourse) seems to suggest that our perceptual categories have a direct counterpart in the physical world, such as in the case of 'illumination' or 'surface', a closer examination reveals that physically defined categories and perceptually defined categories do not match, and that, with respect to the functioning of the perceptual system, the occurrence of the former is neither necessary nor sufficient for the occurrence of the latter. The way we perceptually segment our world does not conform to the way we divide up things in the world when we are doing physics (an observations that Ludlow, 2003, p.150 terms a "type mismatch"). 
Although naïve realism already founders in the face of the most elementary scientific facts, say about the properties of our sense organs, it intellectually expresses some of our deepest convictions about the mental activity of perceiving, namely being in direct touch with a mindindependent world. These convictions - which Hume, in his An Enquiry Concerning Human Understanding, § 118, regarded as "a natural instinct or prepossession" - are so deeply entrenched in our conception of the world and our interactions with it, that it is hardly surprising that they exercise a continuous impact on perception research (where they are particularly explicit in current versions of Bayesian frameworks for perception, cf. Mausfeld, 2002). ${ }^{1}$

Our built-in conviction that perception represents the way the external world is constitutes itself a functionally important achievement of our brain, and thus ultimately has itself to find a place in an explanatory account of our mental capacities. It becomes detrimental, however, when it is, with respect to perception theory, illegitimately transferred to scientific enquiry. In this case, it goes along with the tendency to use categories of the output for a description of the input. The predisposition to use physical categories from common-sense taxonomies, and thus from the output of the perceptual system, as independent descriptions of the physical world that allegedly need to be 'recovered' from the sensory input in the process of perception pervades perceptual psychology. By thus mistaking output categories for input categories, the proper explanatory task of perception theory is trivialised. This was emphasised particularly by the Gestaltists and by Albert Michotte. Michotte was exceptionally sensitive to the problem of meaning in perception. His experimental work led him to acknowledge that "our sensory experiences ... are infinitely richer in content than could ever have been anticipated" and that underlying such achievements "seems to be a kind of 'prefiguration' of abstract concepts, the mental 'categories' of substance, reality, and causality” (Michotte, 1954/1991, p. 44/45).

\footnotetext{
${ }^{1}$ Corresponding common-sense intuitions are also severely impeding an adequate theoretical understanding of 'intentional phenomena'. As Barry Smith (1994, p. 40; cf. also Moran, 1996) noted with respect to Brentano’s conception: "One will find no coherent interpretation of Brentano's principle of intentionality so long as one remains within the framework of our usual, commonsensical notions of both the mind and its objects." Brentano's doctrine of intentionality "involved a radical overhaul of our common-sense conception of the veridical intentionality of our normal acts of seeing and thinking" (Smith, 1994, p. 132). According to Brentano, "the phenomena of light, sound, heat, spatial location and locomotion which [the natural scientist] studies are not things which really and truly exist. They are signs of something real, which, through its causal activity, produces presentations of them. They are not, however, an adequate representation of this reality, and they give us knowledge of it only in a very incomplete sense. We can say that there exists something which, under certain conditions, causes this or that sensation. We can probably also prove that there must be relations among these realities similar to those which are manifested by spatial phenomena of shapes and sizes. But this is as far as we can go" (Brentano, 1874/1995, p. 19; 1874/1973, p. 28). As Smith (1994, p. 254) argued, Brentano "has no room for physical things in the standardly accepted sense. He sees the world rather as a kind of sensory surface, capable of being partitioned into constituent sub-surfaces more or less ad indefinitum. To some of the sub-surfaces thereby generated a certain 'thing-character' may then be subjectively imputed." Consequently, on Brentano's conception, "the primary object of the intentional reference cannot exist outside the mind" (Albertazzi, 2006, p. 279). However, most of Brentano's followers, and in fact the reigning perspectives in contemporary philosophy of mind, have decisively departed from Brentano in favour of conceptions of 'intentional reference' that preserve our realist common-sense intuitions.
} 


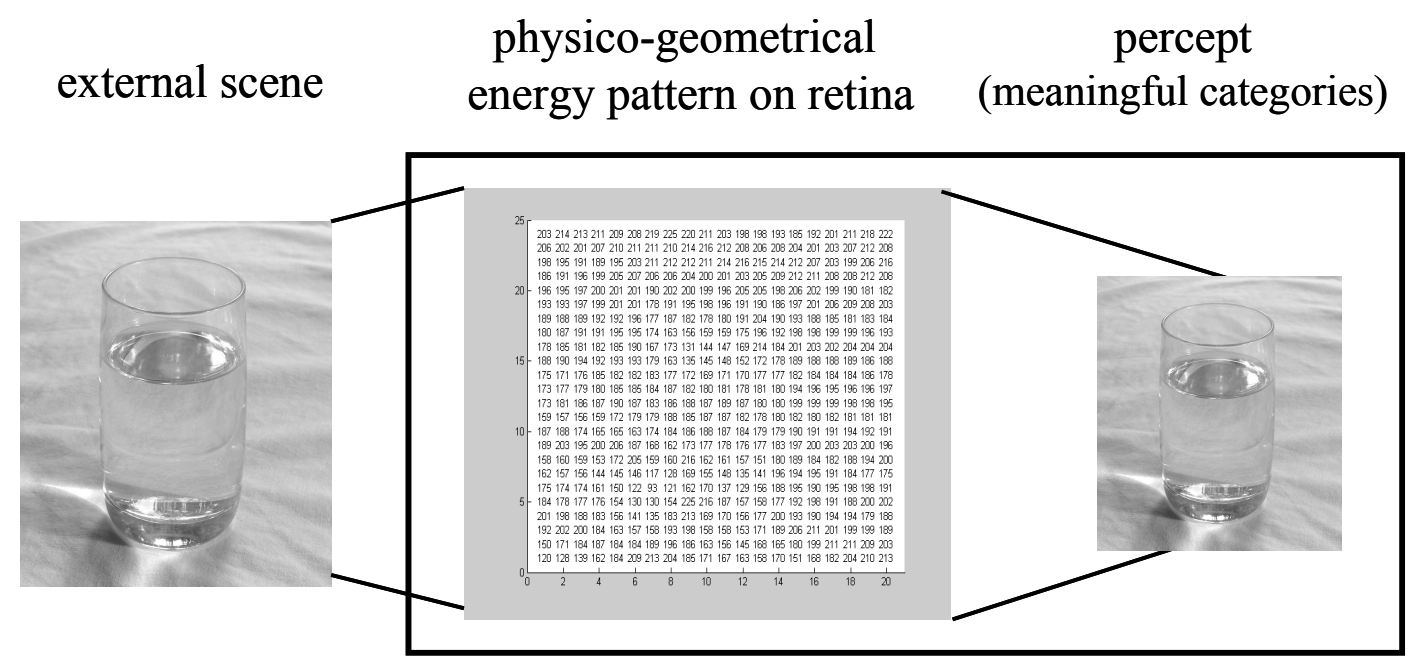

Figure 3: How the explanatory gap is trivialised in common-sense conceptions of perception

In order not to succumb to our common-sense preconceptions of perception and for theoretical reasons given in more detail later, we should avoid, in the present context, any reference to an external scene, and thus to the potential external causes of the sensory input, and rather confine the formulation to the actual input of the biological system under scrutiny. The Fundamental Problem of Perception Theory amounts then to the question: On the basis of what principles can the perceptual system generate, given a specific physical spatio-temporal energy pattern as input, an output that is organised in terms of meaningful categories, as depicted in figure 4? The task of perception theory thus is to identify the internal structure, i.e. the complex data types and computational principles, of a biologically given computational system, on the basis of which the system can generate perceptually meaningful semantic distinctions given certain sensory inputs.

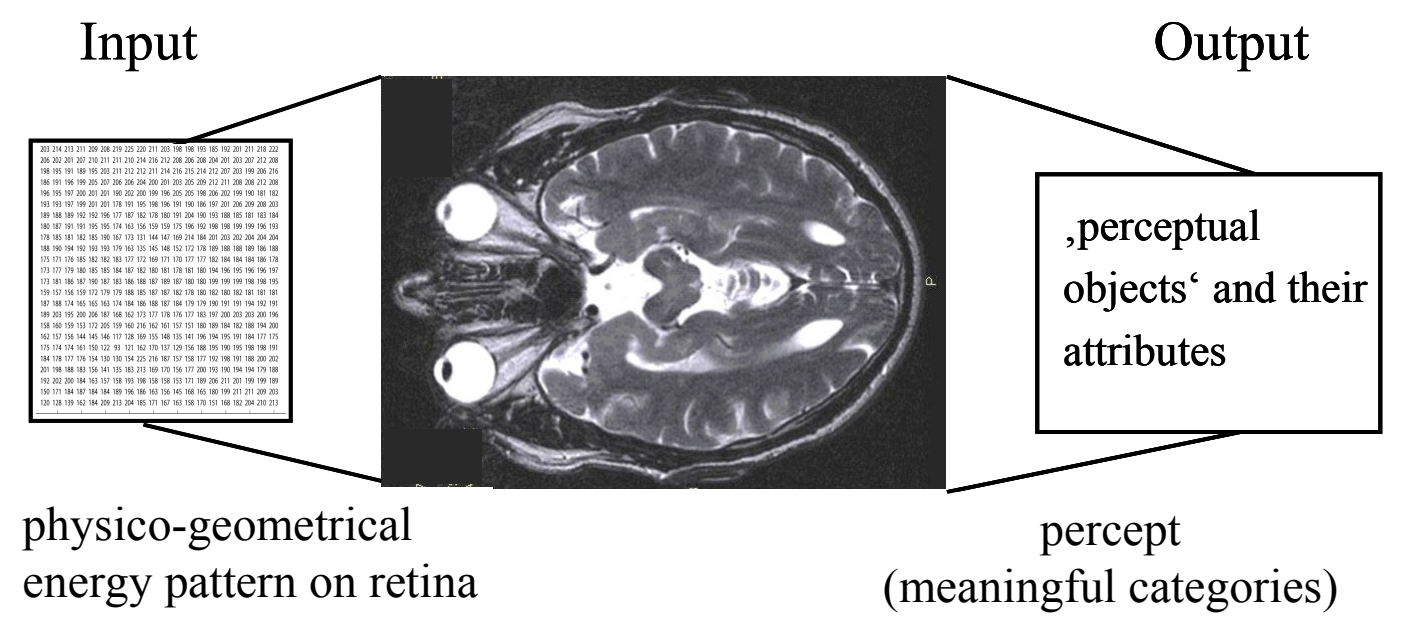

Figure 4: The Fundamental Problem of Perception Theory 


\section{The Standard Model of Perceptual Psychology}

Perceptual psychology and psychophysics, in most of their currently prevailing approaches, have framed their enquiries in a way that either by-passes or trivialises altogether the Fundamental Problem of Perception Theory. Their underlying conception of perception condenses in what can be called the sensation-perception model of perception. This conception is grounded on a distinction between sensations, as the 'raw material' of experience, on the one hand, and perceptions, which were typically conceived of as referring to objects in the external world. A distinction of this kind, as advanced by Spencer, James, Wundt or Helmholtz, pervades, in various forms, perceptual psychology. Sensations and perceptions were generally held to "shade gradually into each other, being one and all products of the same psychological machinery of association" (William James, Principles of Psychology, Ch. XIX), or of some other inferential machinery. The sensation-perception model of perception thus conceives of perceptions as a hierarchy of processing stages within the same set of sensory data types by which the sensory input is transformed into the meaningful categories and distinctions, namely the 'perceptions', whose meanings derive from what they refer to in the external world.

The sensation-perception model, in its many guises, became what may be called the Standard Model of perceptual psychology and psychophysics. According to this model, the process of perception can essentially be described as subsequent stages of 'information' processing by which the sensory input is successively transformed into the percept, as illustrated (following Neisser, 1976, p. 17) in figure 5. (As to the concept of information underlying this scheme, Jackendoff (1987, p. 38) noted that "sometimes one gets the impression that information is to be thought of as a sort of abstract liquid that is poured from one receptacle into another, that is filtered free of unwanted detail, and that overflows into forgetfulness if the receptacle into which it is being poured is already full.") Although the notion of 'information', as employed in the context of corresponding conceptions of perception, is notoriously vague and beclouds the actual problems involved, this Standard Model of Perception is mostly regarded in current orthodoxy not as a model but as a self-evident truth.

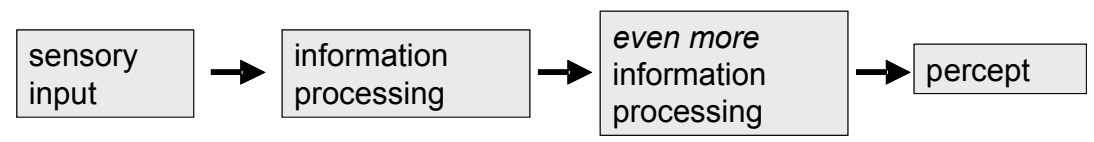

Figure 5: The Standard Model of Perception

More specifically, it is presumed that the sensory input provides information about elementary sensory qualities, such as colour and motion, and that by so-called lower level processes these elementary qualities are glued together by some associative or inferential machinery (as depicted in fig 6). The remaining gap to the percept is then bridged by what is usually referred to as 'cognitive processes' or 'higher-order' processes, which are assumed to account for everything that cannot be explained by the so-called lower-level processes. 


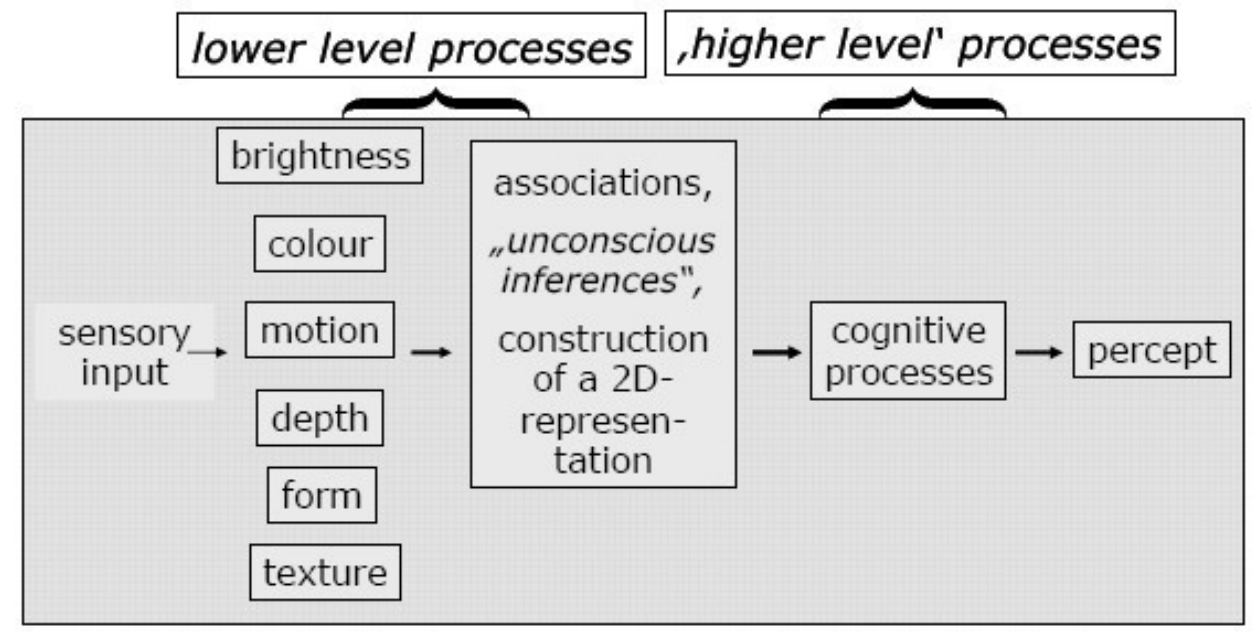

Figure 6: More detailed description with respect to vision of the Standard Model of Perception

There are basically two ways open for interpreting these schemes of perceptual information processing, both equally fatal. On a weak interpretation, the arrows indicate, in a loose colloquial manner, some temporal sequence, leaving the kind of relations between the boxes, particularly in the last step, entirely unspecified. On a stronger interpretation the arrows are understood as indicating consecutive steps of mathematically definable transformations of the sensory image by which the output of a previous step is transformed to yield the input for the next transformation, by which finally the percept is yielded. While the first interpretation completely by-passes the Fundamental Problem of Perception Theory, namely to explain how semantic perceptual categories can arise from a stimulation by physical spatio-temporal energy patterns, the second interpretation amounts to an alleged solution of this problem that is deeply flawed already on conceptual grounds, for reasons addressed above.

The key flaw of the Standard Model is that it dodges, by its very conceptualisation of perception, an essential task of perceptual research, viz. the identification of the internal conceptual structure of perception and of the symbolic objects to which the computational procedures of the perceptual system apply. The Standard Model is, moreover, not even aware of this explanatory deficit because it borrows semantic distinctions, such as 'surfaces', 'shadows', or 'illumination', tacitly from the output of the perceptual system and uses them for a description of the input (particularly when the Standard Model is supplemented by an inverse optics approach of ,recovery of world structure'). Thus, the problem of perceptual semantics has not even been recognised by traditional approaches as a serious theoretical challenge because it is concealed precisely by one of the eminent achievements of our brain, viz. the externalisation of its own semantic categories into what we regard as the external world.

The gross inadequacy of the Standard Model's way of framing the explanatory task of perception theory has been pointed out since the $17^{\text {th }}$ century. By corresponding enquiries, it became evident that the problem of perceptual meaning cannot be resolved by deferring the explanat- 
ory duty to the sensory information. Not even the core notion of a 'perceptual object' can be derived, by whatever mathematical machinery, from the sensory input. Hume was well aware of this problem and noted, in his Treatise of Human Nature (Book 1, Part IV, sec II), that the senses "give us no notion of continu'd existence, because they cannot operate beyond the extent, in which they really operate. They as little produce the opinion of a distinct existence, because they neither can offer it to the mind as represented, nor as original... We may, therefore, conclude with certainty, that the opinion of a continu'd and of a distinct existence never arises from the senses." Therefore it has generally been noticed since the $17^{\text {th }}$ century that there was an explanatory gap to be filled. Michotte was particularly cognisant of the theoretical challenge that the "remarkable case of phenomenal permanence" (Michotte, 1954/1991) and the underlying notion of the identity of an object over time and over various classes of transformations poses for perceptual psychology (cf. Spelke, Gutheil \& Van der Valle, 1995; Casati, 2005; Scholl, 2007). Perception theory has to provide an explanatory framework for these kinds of basic phenomena. However, the explanatory task to come to terms with the most fundamental theoretical notion, viz. that of a 'perceptual object', is dodged by deriving this notion from a allegedly 'corresponding' notion of an 'external world object' and by placing the explanatory burden on experience and some inferential machinery. These externalist approaches to the problem of perceptual meaning, which historically were advanced notably by Hume and Helmholtz, became to dominate perceptual psychology. In the context of the Standard Model, various types of mechanisms have been proposed, by which sensory experiences can allegedly be transformed into perceptual categories. For instance, Hume assumed that mechanisms "of compounding, transposing, augmenting, or diminishing the materials afforded us by the senses and experience" (An Enquiry Concerning Human Understanding, Section II) were at the core of our mental capacities; Helmholtz placed the explanatory burden on experience-based "unconscious inferences", and more recent computational approaches regarded at the core of perception some kind of sensory data-based inferential processes of a recovery of 'world structure'. The apparent attractiveness of these conceptions of perception predominantly derives from the fact that they accord with our common-sense conceptions of perception. They are, however, profoundly inadequate already on conceptual grounds. Later developments and insights into what can be achieved by inductive procedures made clear that no general inductive machinery, however powerful, can derive from the sensory input, and thus, more generally, from experience, the kind of internal conceptual structure that is explanatorily required (unless it is already itself based on a conceptual structure as powerful as the one to be inferred). However powerful the inductive machinery is assumed to be, there is no way to arrive at symbolic objects that are logically more powerful in the sense that there structure is not expressible in the logical language in which we describe the bases of the inductive procedure (cf. Fodor, 1980). Since essentially no perceptual object or attribute of our perceptual system is definable in the logical language by which we describe the physical energy pattern that constitutes the sensory input, the internal structure underlying perceptual meaning cannot be attained by inductive procedures (unless we surreptitiously describe the input in terms of the yet-to-beexplained output categories). All the same, prevailing thinking in perceptual psychology and psychophysics is marked by its deep-rooted commitments to a more or less naïve realist metaphysics and an empiricist theory of mind - as Köhler (1947, p. 140) noted, perceptual psycho- 
logists are "so fond of their empiricist convictions". Such detrimental influences of commonsense conceptions are an important factor for why large parts of the field have turned into a data-driven and hence non-progressive enterprise.

In the history of perceptual psychology, the strongest critique of the Standard Model had been advanced by Gestalt psychologists, on the basis of accumulating empirical evidence. They furthermore recognised that the Standard Model's emphasis on issues of processing results from mistaking the explanatory task of neurophysiology for the explanatory task of perceptual psychology, and thus from conflating different levels of analysis. However, the Standard Model is, despite its utter inadequacy, almost taken as a truism in much of current perceptual research. It is inherent to truisms that they obviate the conception of any alternatives. Accordingly, the alternative conceptions of perception that fuelled Gestalt psychology, Michotte's "experimental phenomenology", or the approaches of many others who recognised how rich the internal conceptual structure of the perceptual system is have been disregarded. This situation appears to be changing in more recent years, during which ethology and various domains of cognitive science, notably perceptual research with babies, have been providing a wealth of experimental findings in support of a radically different conception. Although the theoretical conceptions underlying these experimental investigations have mostly remained implicit, the distinguishing features of these ways of framing the task of perception theory are clearly discernible. Before I will attempt to characterise some core elements of the emergent theoretical framework, I shall, in the following section, deal with a more general, methodological issue, namely with the question of what can be regarded as an appropriate level of idealisation for dealing fruitfully with the problem of meaning in perception theory.

\section{The problem of the internal semantics of the perceptual system}

A decisive feature of the above formulation of the Fundamental Problem of Perception Theory is that it singles out a specific level of analysis as particularly appropriate for gaining theoretical insights into the nature of perception. This level of analysis pertains to an investigation of the internal principles of a specific biological subsystem. It abstracts away from aspects with which other levels of analysis are concerned, for example aspects pertaining to neural implementation and physical basis, or to evolutionary development and adaptive function.

It is a matter of course that the assumption that the perceptual system qualifies as a subsystem of the brain that can, by standard methodological practices of idealisation and abstraction, be studied in isolation, in no way implies the denial of dependencies with other systems. With respect to rational enquiry, the question is not, how in reality things are related to each other. The nature and functioning of the perceptual system is related to and dependent on various aspects of reality like its phylogenetic development, on the metabolic system, the immune system, or a great variety of other internal computational systems, or on the physics of the 
brain. The question rather is what constitutes an appropriate level of idealisation for successful explanatory frameworks of perception.

From our common-sense intuitions, an abstraction that focuses on the internal principles of the system involved appears to be rather odd because it discards most of what we usually consider important questions about perception. In the context of the natural sciences, however, such an abstraction, which is in line with general methodological principles of multi-layered analyses of biological systems (e.g. Tinbergen, 1964), disburdens the explanatory task of perception theory from issues that do not promise to advance theoretical understanding of the internal principles of the system under scrutiny.

Among the issues that should be considered as external to the explanatory task of perception theory proper are also issues that pertain to levels of analyses employed by ecological physics or by functionalist evolutionary biology. These fields are primarily concerned with, e.g., understanding the kinds of physical regularities of the external world that are taken advantage of by the perceptual system, with functional and adaptive aspects of the perceptual system, or with its evolutionary history. To be sure, the perceptual system, as other computational or non-computational biological systems, has, in its evolutionary development, taken advantage of external physical regularities in the sense that the way internal mechanisms work is moulded by or even determined by specific external physical regularities. From this however, it does not follow that considerations about adaptive purposes or about the 'proper' external objects of perception figure in explanatory accounts of the internal principles by which the perceptual system generates its outputs on the basis of specific inputs. As in the case of other biological systems, an understanding of the internal functioning of the perceptual system neither rests on a diachronic analysis of its selectional history nor on considerations of which physical entities should be regarded as the 'true' or 'proper' antecedents of the sensory input (aside from heuristic purposes and our ordinary or meta-theoretical talk, in which such enquiries are inevitably embedded). Needless to say, perception must structurally mirror or at least not contradict biologically relevant aspects of the external world. This, however, is hardly an insight but rather simply rephrases from a functional point of view the kind of mental phenomena that have been singled out as an object of enquiry. From it, it does by no means follow that categories or attributes of perception are categories or attributes of the external world: Even if perception would mirror not even in a single case the true manner of being of the external world (whatever that is supposed to be), it still could provide a coupling to biologically relevant structural aspects of it.

Therefore, the above formulation of the Fundamental Problem of Perception Theory in particular dispenses with considerations about what might be the 'proper' objects in the external world that are causally responsible, among the infinite set of potential causal antecedents, for the sensory input. Each sensory input that gives rise to a specific percept can be physically produced in many different ways (think of an object on a CRT screen or in a virtual reality setting). Because it is, for the explanatory purposes of perception theory, immaterial what the distal causes of a certain sensory input are, any reference to the potential distal causes of the 
sensory input is extrinsic to perception theory. Consequently, notions such as 'perceptual error', 'veridicality', 'reference' or 'proper function' have no place in explanatory accounts of the functioning of the perceptual system, and there are no explanatory lacunae in perception theory to be filled by introducing these notions. Particularly the notion of 'perceptual error', which is a distinguishing element of our ordinary discourse about perception, is of no avail for perception theory (cf. Mausfeld, 2002, Mausfeld, in press a).

Perception theory rather attempts to theoretically understand the perceptual system and to identify its abstract internal principles by which it generates, given specific physical spatiotemporal energy patterns as inputs, outputs that are organised in terms of meaningful categories. The semantic categorisations that the perceptual systems supplies to subsequent systems are not determined by 'corresponding' properties of the external world. Rather, they are individuated by the kind of data formats and by the computational machinery with which the perceptual system is biologically endowed. Perception theory thus places the explanatory burden for an understanding of perceptual categories on the internal structure of the biological system under scrutiny. While the problem of meaning is mostly unrecognised or dismissed as almost trivial in our common-sense conception of perception due to its built-in externalisation of 'perceptual meaning', perception theory considers this problem as being at the core of its explanatory task. I will refer to it as the problem of the internal semantics of the perceptual system.

Investigations of the internal semantics of the perceptual system aim at identifying the data formats, and thus the symbolic objects, on which the computational procedures of the perceptual systems are based. The form or logical structure of these symbolic objects and the structure of their interrelations define the core 'perceptual ontology' of the perceptual system and the type of computational principles that can be applied to these objects. ${ }^{2}$ The internal semantics therefore exhaustively characterises the 'world' as conceived of by the perceptual system, and hence the kinds of 'perceptual objects' of the perceptual system and the way they are related. The internal semantics of the perceptual system does not capture percept-world relations but only the relation between internal perceptual objects. While the external semantics of perception, i.e. acts of referring to entities in the external world, is dependent on the complex interaction of the perceptual system with higher interpretative systems, and applies to the level of the entire organism, the internal semantics is an intrinsic property of the perceptual system. Because of this, the internal semantics captures those aspects of 'perceptual meaning' that are amenable to abstraction and idealisation and thus can reasonably be regarded to be within the reach of naturalistic enquiry.

Confining semantic issues to intrinsic properties of the system under scrutiny is often referred to as an internalist approach to meaning. ${ }^{3}$ From the perspective of our common-sense intuitions of perception, an internalist approach disregards what we, in ordinary context, consider

\footnotetext{
${ }^{2}$ Corresponding intuitions have a long history in perception theory. They also find their expression in Brentano's conceptions. With his psychology from an empirical standpoint, Brentano intended to develop, as Albertazzi (2006, p. 335) aptly put it, a "general theory of inner form."
} 
as the most important element of 'perceptual meaning', namely our acts of referring to 'objects in the external world'. From an internalist view, however, this capacity depends on the intricate interpretative capacities brought forth by the integrative action of the entire system, i.e. of persons. Hence it does not promise to provide a deeper theoretical understanding of intrinsic properties of the specific system under scrutiny, i.e. of the internal principles of the perceptual system.

What appears, from our ordinary perspective, to be an illegitimate stricture on the study of perceptual meaning turns out to be an eminent methodological advantage for the explanatory task at hand. An internalist approach emphasises the fact that what we refer to in perception as 'objects in the world' are in fact mental objects, and thus directs attention to the central question of what constitutes a 'perceptual object'. Furthermore, it makes incontestably clear that explanatory demands compel us to ascribe a rich internal structure to the system, not unexpected for a complex biological system. Since the perceptual system is regarded as a computational system, this means to ascribe to it a rich structure in terms of its internal data formats and computational principles.

Internalist approaches to perception have a long and fruitful tradition in the field. The underlying conceptual frameworks were first laid out during the $17^{\text {th }}$ century. They found their most sophisticated and strongest expression in Descartes' sign theory of perception and his idea of a 'semantic relation' between the sensory input, on the one hand, and the conceptual forms by which the perceptual system is endowed, on the other hand (cf. Yolton 1996, p. 73ff; Gaukroger 1995, p. 287).

An internalist approach has also been, implicitly or explicitly, at the basis of ethological enquires (e.g. Lorenz, 1941, 1982; Tinbergen, 1963; Gallistel, 1998). Ethological investigations into the perceptual capacities of different species, which have not been encumbered by our common-sense intuitions about perception, have yielded fruitful explanatory frameworks for dealing with issues of the internal semantics of different perceptual systems. Internalist approaches have also yielded copious and fruitful insights during the history of perceptual psychology. It has long been noted that "the visual system populates 'the world' with all sort of 'objects' that have no physical reality" (Jackendoff, 2002, p. 308; cf. Köhler, 1947, p. 157). Think, for example, of perceptual objects associated with figure-ground segmentations, perceptual groupings, or amodal completions. These observations and phenomena constituted the focus of Gestalt psychology, which was firmly rooted in an internalist perspective. (As is known, the theoretical frameworks of the Gestaltists were critically deficient. However, one has to bear in mind that neither the Gestaltists nor previous thinkers had the conceptual apparatus provided by the theory of computation at their disposal. They therefore could not appropriately formulate the abstract issues involved but rather had to couch their insights in their

\footnotetext{
${ }^{3}$ Brentano, who famously held that the true method of philosophy is nothing other than that of natural science (,, Vera philosophiae methodus nulla alia nisi scientiae naturalis est."), also exhibits a strong internalist inclination. Cf. Albertazzi (2006, p. 111): "If we look at the relation between the thought and the primary object in Brentano's doctrine, we must remember that the real thing to which this thought refers cannot exist outside of the mind."
} 
own and often idiosyncratic terms which served as makeshift.) The Gestaltists were also aware that our perceptual objects, i.e. the symbolic objects of the perceptual system, cannot, in any meaningful sense, be understood as representations of 'corresponding' physical categories. However, this insight has almost been lost in perceptual psychology, which, due to built-in preferences for an externalist conception of perception, routinely employs this term 'representation' in the sense of a world-mind-mapping. ${ }^{4}$

In other areas of cognitive science, notably linguistics and enquiries into lexical semantics, internalist approaches yielded highly fruitful explanatory frameworks for various domains of mental phenomena. The most prominent advocate of an internalist approach is Chomsky (e.g. 1966, 1996, 2000), who has pioneered and elaborated it as a general research programme for the study of mental phenomena and adamantly defended it against externalist conceptions.

Perception theory, as the attempt to gain theoretical insight into the principles underlying the mode of operation of the perceptual system, has first and foremost to address the question of what constitutes the minimal meaning-bearing elements and thus the 'perceptual objects'. In line with the wealth of empirical observations and phenomena that have been accrued in the history of the field, perception theory acknowledges the fact that perceptual meaning can not be derived from the sensory input or from a description of the external world, no matter how sophisticated the mathematical apparatus underlying such attempts is. The available evidence also shows that the biologically given internal semantics of the perceptual system is multifariously rich and variegated, as well as highly intricate and unexpected. Its features are highly idiosyncratic with respect to our physical descriptions of the external world, and often strongly deviate from our ordinary intuitions and expectations. They can only be identified by empirical investigations of the internal structure of perceptual objects and their attributes.

In the next section, I will attempt to expose a few very general principles of perception that I believe to be both well-motivated and empirically well-supported by the corresponding work in perceptual psychology, ethology, research with babies and internalist enquires into other domains of the cognitive sciences.

\footnotetext{
${ }^{4}$ The widespread acceptance of this term, which has yielded tremendous philosophical confusion, historically seems to have been enhanced by the French translations of Descartes' profound and deep analyses of perception. Descartes had used, e.g. in his Third Meditations, the Latin verbs 'exhibeo' and 'repraesento' interchangeably, in the sense of something that is internally presented to the mind. In seventeenth-century French both Latin verbs were translated by 'representer', so that the meaning of the term shifted from denoting a predominantly mindinternal presentation to denoting a mind-world relation. Although Descartes had an internalist meaning in mind, the French translation tacitly was guided by our ordinary preference for an externalist interpretation of the term.
} 


\section{On the functional architecture and the conceptual forms of the perceptual system}

At the core of the theoretical picture that can be abstracted and distilled from the corresponding empirical ${ }^{5}$ and conceptual work is the distinction between (at least) two different subsystems of the perceptual system. Such a distinction with respect to the functional architecture of the perceptual system is required in order to explanatorily account for the "wide gulf between sensory stimulus and percept" (von Szily, 1921, p. 971). As mentioned before, there is, within the mathematical language employed by physics, no mathematical machinery, however powerful, by which the specific conceptual structure underlying the organisation of our percepts can be derived from the physical sensory input. The abstract concepts underlying perception and the "mental categories of substance, reality, and causality" (to use Michotte's words) can only be expressed in a natural way in a logical language that is strictly richer and more powerful than the logical language appropriate for a description of the sensory input. In other words, these abstract concepts are neither reducible to nor expressible by the concepts of the logical language in which we describe the sensory input. Hence, there is no way to derive them within that language (as much as, say, the biological concepts of the 'cell' cannot be defined within a purely physical language, or as mental concepts cannot be defined by or derived from neural ones).

Needless to say, it is exceedingly difficult to formulate in a precise manner a corresponding distinction between a computational subsystem whose data formats can be expressed in the same logical language that we use to describe the input, and computational subsystems whose data formats are not expressible in this logical language. Nonetheless, a distinction of this kind is at the root of a venerable and fertile intuition that, in various guises, pervades the history of perceptual psychology. Using the terminology employed here, this intuition amounts to the idea that the 'nature' of conceptual forms is not exhausted by their extensional descriptions in terms of sensory inputs or sensory codes by which these forms can be elicited but rather requires an autonomous level of analysis (employing a suitably rich logical language). With respect to a computational architecture, this idea entails the requirement to make precise

\footnotetext{
${ }^{5}$ The contributions of Gestalt psychology and of Michotte may vicariously stand for the overwhelmingly rich amount of findings of perceptual psychology that are in support of this theoretical picture. With respect to colour, I have given in Mausfeld (2003) a more detailed account of relevant findings. From the plenitude of relevant experimental findings that have been contributed by other domains, I will only mention here, in a rather eclectic way, a few examples. Examples for pertinent contributions from developmental psychology are: Baillargeon (1993), Bonatti, Frot, Zangl \& Mehler (2002), Hamlin, Wynn \& Bloom (2007), Spelke (2000), Spelke, Gutheil $\&$ Van der Valle (1995), Trevarthen (1998), or Wang \& Spelke (2002). From ethology, I will mention by way of example Gallistel (1998), Hare, Call, Agnetta \& Tomasello (2000), Hauser, Pearson \& Seelig (2002), Premack \& Premack (1995, 2003), Santos, Hauser \& Spelke (2001), or Wehner (2003). Evidence that is of relevance with respect to this conception also comes from linguistics (Chomsky, 2000; see also, e.g., Pustejovsky, 1995; Yang, 2002) and from neurophysiology (e.g. Caramazza \& Shelton, 1998). Furthermore, the conception outlined is consonant with well-supported broader meta-theoretical perspectives on the nature of mental phenomena (e.g. Strawson, 2003; Hinzen, 2006). To be sure, no single experiment and no set of experiments can ever be regarded as conclusive evidence for the perspective outlined or any other theoretical framework. My confidence in this conception derives as much from theoretical considerations as from empirical findings. Furthermore, it is, as always in the natural sciences, fortified by the fact that this conception is suggested by a theoretical convergence of quite different disciplines.
} 
how to conceive of computations between systems whose internal computations are based on different sets of primitives, respectively.

Accordingly, explanatory needs require us to distinguish (at least) two different subsystems of the perceptual system (or two different levels of abstraction, which will be identified here with actual idealised subsystems) that mediate between the sensory input and its output. The defining difference between these two subsystems is the expressive power of the logical language by which their respective data formats can be defined. At the front-end of the perceptual system, we can single out a subsystem whose operational mode can be completely described in terms of the physico-geometrical language in which we describe the sensory input. Such a logical language is, however, incapable of expressing the core concepts underlying the output of the perceptual system. Therefore, we have to postulate a second system which is based on a more powerful logical language and whose data formats and symbolic concepts yield all the semantic distinctions that characterise the output of the perceptual system. I will refer to these two systems as Sensory System, on the one hand, and Perceptual System, on the other hand (this distinction differs fundamentally from the one associated with the Standard Model). I shall use the generic term 'perceptual system' to refer to the system that is composed of the Sensory System and Perceptual System as characterised here. The general functional architecture of the perceptual system can be illustrated by figure 7 .

SENSORY SYSTEM

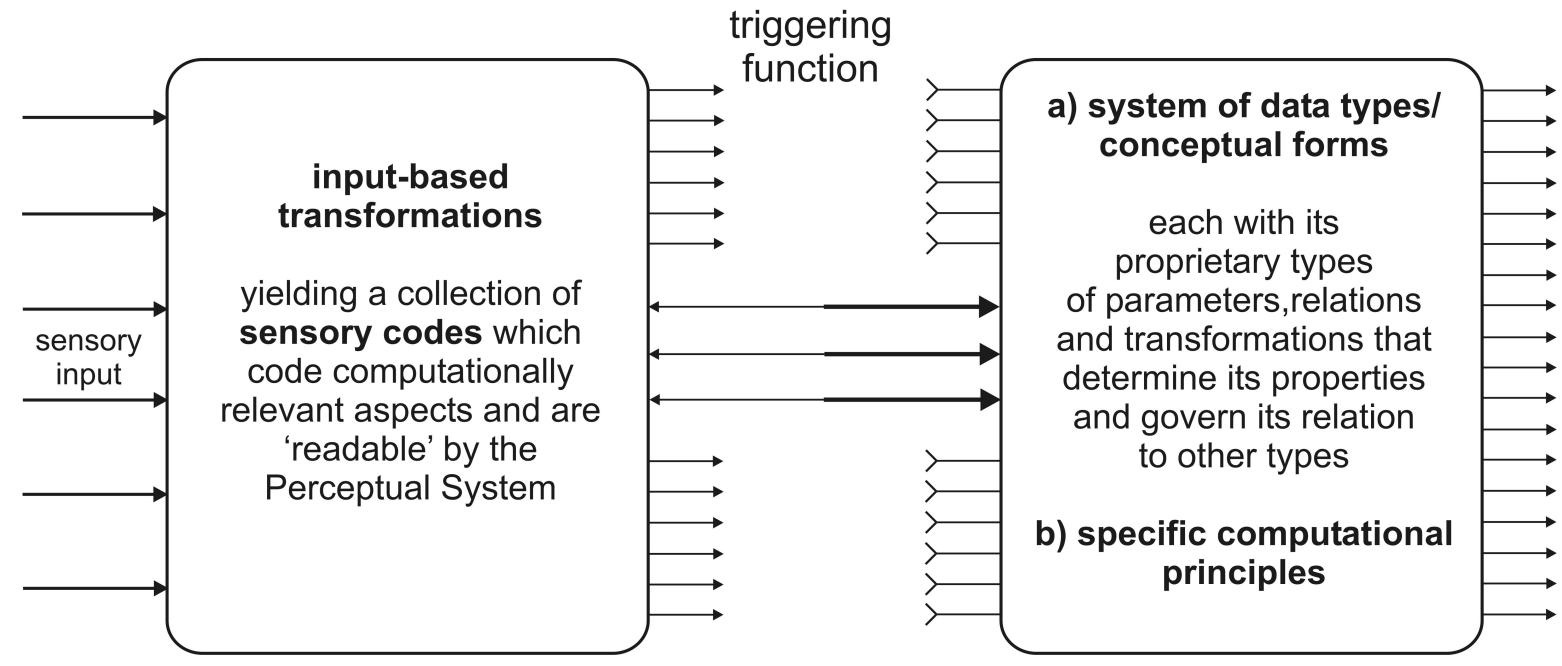

Figure 7: Functional architecture of the perceptual system

The Sensory System deals with the transduction of physical energy into codes that fulfil the structural and computational needs of the Perceptual System and that are, at its interface with the Perceptual System, 'readable' by it. By definition, the data formats on which the computations of the Sensory Systems are based are definable in terms of the same physico-geometrical language by which the sensory input can be described. The operations of the Sensory System 
are, accordingly, purely sensory-based transformations such as filtering and convolutions, calculation of certain derivatives of luminance distributions, gain control operations, calculations of global statistical features, or any other mathematical operation on the sensory input or on codes obtained from such operations. Considerable insights have been gained by sensory physiology and psychophysics into the operations of the Sensory System, which has been the proper object of enquiry of these fields. The Sensory System pre-processes the sensory input, in terms of a rich set of input-based symbolic data types, in a way that is dynamically interlocked with the specific requirements of the Perceptual System. A more detailed analysis of it would in turn necessitate a distinction of different levels of abstraction (which extend from transduction codes and simple transformations thereof to highly abstract codes in term of internal symbolic objects of the Sensory System).

The conceptual apparatus of the Sensory System is itself highly hierarchically organised. At the top of this hierarchy and thus at the level of its interface to the Perceptual System, this apparatus particularly includes abstract concepts that are highly adapted for the demands of the Perceptual System. Among these concepts are ones pertaining to 'texture', 'connected region', 'optical flow', various types of 'edges' or 'junctions' etc., or, in Marr's terms, notions by which a "2D representation" can be characterised.

Among the expressions of the Sensory System are also those that have been traditionally referred to as 'cues' or 'signs'. Cues or signs are considered to be those features of the sensory input that supposedly stand for and convey information about properties of external objects or relations. Accordingly, they have been regarded, since Alhazen and Helmholtz, as providing the base of evidence in inferential accounts of perception. On such conceptions, the inferential procedures have to embrace methods of 'cue integration' and 'cue combination' because each single cue is regarded as ambiguous, incomplete and of limited accuracy with respect to the properties it is supposed to represent. Although the cue concept is among the most popular ones in perceptual psychology, it hovers, in its ordinary usage, theoretically in the air. It is again based on mistaking the output of the perceptual system for its input. It takes for granted what actually has to be investigated, namely the conceptual structure in which it has to be grounded. Cues do not bear any meaning by themselves. Rather, the notion of a cue is conditional upon the conceptual structure by which cues are invested with meaning. Thus, the notion of a cue or sign cannot be detached from explicitly specifying the entire conceptual system with respect to which something can function as a cue. Speaking of cues or signs prior to a specification of the conceptual structure, by which cues or signs are conveyed their meaning, only makes sense within our ordinary discourse about perceiving (which takes for granted what actually has to be part of an explanatory framework of perception theory). In the context of perception theory, however, we need to first identify the core structural properties of the Perceptual System before we can reasonably refer to certain symbolic objects and their attributes, relations etc. of the Sensory System as cues or signs. It is important to realise that such 
notions refer to relations that are entirely mind-internal. What is signified by a cue or sign is not an external object but a concept of the Perceptual System. ${ }^{6}$

Correspondingly, taking of cues as being ambiguous or incomplete refers to entirely internal relationships between a whole system of cues provided by the Sensory System, on the one hand, and the conceptual forms of the Perceptual System, on the other hand. The intricate issues involved cannot be dodged by surreptitiously using the semantic distinctions that are generated by the Perceptual System for a description of its input. With respect to perception theory, the main explanatory burden has to be placed on enquiries into the specific structure of the conceptual forms of the Perceptual System and their interrelation.

The structure of the data formats and conceptual forms underlying perception have as yet, however, not been the target of systematic investigations in perceptual psychology. Rather, perceptual psychology has largely bracketed the problem of perceptual semantics by focussing on perceptual processing with respect to data formats that either are defined by neurophysiological magnitudes or are presumed to mirror what we, in our quotidian description of the external world, consider to be external world categories (such as surfaces, illuminations, shadows, trees, animals, etc.). Among the few conceptual forms whose structure and triggering conditions have been investigated more systematically are the core ones pertaining to 'perceptual objects'. These studies have already yielded significant theoretical insights into the constitution of a 'perceptual object' by a spatio-temporal continuity, as paradigmatically illustrated by Michotte's “tunnel effect” (cf. Burke, 1952; Flombaum \& Scholl, 2006), and by internal principles of cohesion and solidity, the study of which has been pioneered by Spelke (e.g. 1990, 2000). Apart from these and a few other exceptions, there is, in contrast to the abundance of studies that deal with aspects of processing, almost a complete absence of experimental or theoretical studies that specifically address the "information structure" (Jackendoff, 1987, p. 39) on which any kind of processing by definition is based. Therefore, we have only the most rudimentary ideas about the specific types of its conceptual forms and

\footnotetext{
${ }^{6}$ Descartes clearly recognised that any reference to 'external objects' in sign conceptions of perception would lead, within scientific enquiry, to conceptual incoherences. Descartes explicitly identified what I refer to as the Fundamental Problem of Perception and the deep explanatory gap that is associated with it. In his attempts to provide, in entirely naturalistic terms, an explanatory framework for bridging this gap, Descartes (1642/1985) formulated a purely internalistic version of a sign theory of perception. Yolton $(1984,1996)$ referred to Descartes' conception as "inverse sign relation", because for Descartes, the physical motion, as expressed by neural activity, is the sign, and what is signified is what is expressed in the percept. (cf. Gaukroger, 1990, p. 24) For Descartes, there are "two reactions operating in perception: the causal, physiological reaction and the signification reaction" (Yolton, 1996, p. 74). The significatory or semantic relation "replaces the causal relation between physical motion and ideas, but the representing relation goes, as it were, outward from awareness" (Yolton, 1996, p. 190). Descartes thus recognised the explanatory need for postulating a 'semantic relation' in perception. "The connection between the signs and innate ideas is clearly more intimate than any causal connection would be, for it is the innate ideas that make the signs what they are, whereas effects can never make causes what they are." (Gaukroger, 1990, p. 25). Descartes intuitions about this 'semantic relation' between what is provided by the senses, on the one hand, and the conceptual forms or "ideas" that give rise to meaningfully organised percepts were far ahead of anything that could be expressed in terms of the conceptual apparatus available at his time. Unsurprisingly, Descartes' vacillating and tentative usage of terms, due to which he is notoriously hard to interpret, mirrors this lack of an appropriate conceptual framework, as provided much later by computation theory.
} 
their structural properties. All the same, we can derive from phenomenological considerations, from findings of ethology, developmental psychology, and more indirectly, from investigations of the structure of the mental lexicon (e.g. Chomsky, 2000; Pustejovsky, 1995; Jackendoff, 2003) conjectures about the types and structural properties of the conceptual forms that define the internal semantics of the perceptual system.

The conceptual forms of the Perceptual System can be understood as those data formats underlying its computations that are 'visible' at its interfaces with subsequent systems and thus can be 'read' by those systems. As in the formulation of the Fundamental Problem of Perception Theory above, I will deal here, by way of example, only with the conceptual forms on which the semantic distinctions and categories of our phenomenal percept are grounded. In focussing exemplarily on the phenomenal output, it is, however, important to be aware that the structure of the conceptual forms of the Perceptual System is only partly visible at the surface of the phenomenal percept. In particular, we do not notice in our phenomenal experience that the conceptual forms involved are highly underspecified by a given input provided by the Sensory System; rather, the systems that use the outputs of the Perceptual System for constructing the phenomenal percept seems to be furnished with specific computational means to fill-in the blanks, as it were, and thus to completely specify, on a certain level, a phenomenal appearance at each moment.

The output of the Perceptual System is not exhausted by the semantic distinctions and categories of our phenomenal percept; nevertheless, the conceptual forms underlying these phenomenal distinctions and categories provide the basis for our perceptual ontology and thus constitute the realm of 'perceptual objects'. 'Among the exceedingly rich set of complex conceptual forms in terms of which we perceive the 'external world' are conceptual forms pertaining to, e.g., 'surface', 'physical object', 'food', 'tool', 'event', 'potential actor', 'self', 'other person', or 'event', with their associated attributes such as 'walkable', 'manipulable', 'edible', 'colour', 'shape', or 'emotional state', and their appropriate relations such as 'causation' or 'intention'. Conceptual forms create, as it were, what we perceive as the world out there. They define the way in which we perceptually make sense of the world. It is a distinctive feature of the conceptual forms of the Perceptual System that they are not tied to a specific sensory channel and moreover cannot be expressed in terms of purely sensory concepts. This has been emphasised ever since the beginning of systematic enquiry into perception, notably by the Gestaltists. Only on the presumptions underlying the Standard Model can one be surprised about the "evidence for vigorous interaction among sensory modalities" (Shimojo \& Shams, 2001). However, the specific qualitative and quantitative type of interaction can provide important theoretical insights into the internal structure of the conceptual forms involved. These conceptual forms are intrinsically transmodal in character (cf. Köhler's, 1947,

\footnotetext{
${ }^{7}$ Remark on terminology: I do not regard the symbolic objects or conceptual forms of the Sensory System as providing 'perceptual objects'. However, certain aspects (such as gradient, texture, line) of the conceptual forms of the Sensory System can also be captured by the Perceptual System. The question as to which aspects are accessible by the conceptual forms of the Perceptual System is intricate and is intimately related to the classical problem of the so-called proximal mode. In present terminology, the Sensory System does not comprise 'perceptual objects' proper.
} 
p. 204, characterisation of the spatial and temporal perceptual relations of 'disturbance', 'beginning', 'edge', 'hole', 'piece', 'part', 'open', 'incomplete', 'proceeding', 'deviating', 'interrupting').

We can conceive of the conceptual forms of the Perceptual System as abstract structures, each of which has its own proprietary types of parameters, relations and transformations that govern its relation to other conceptual forms and to sensory codes. Furthermore, the available experimental evidence (cf. footnote 2) indicates that the Perceptual System can be regarded as a hierarchically organised typed domain (of possibly unlimited depth). Thus, its symbolic objects belong to different classes of data types, each of which is characterised by specific structural properties and by the type of computations that are applicable to it. ${ }^{8}$ With respect to the conceptual forms considered here, each type defines a set of perceptual objects with uniform behaviour which, in our ordinary language, normally can be named (e.g. 'surface', 'illumination', 'artefact', 'animal', 'mykind'). The hierarchy of types constitutes the biologically built-in 'knowledge' about 'entities' and 'situations' and their properties of our perceptual world, and about our potential 'perspectives' on these. The Perceptual System can therefore be understood as a self-contained system of 'perceptual knowledge', which is coded in the structure of its conceptual forms. The meaning of these conceptual forms is entirely determined by their intrinsic structure and their systematic relations to each other, i.e. by the internal semantics of the Perceptual System. Also, the Perceptual System can reasonably be assumed to have its own computational principles, in particular ones that pertain, e.g., to an evaluation metric, to the satisfaction of internal constraints, or to a more global coherence. In particular, perceptual phenomena suggest that it possesses a rather idiosyncratic evaluation metric (as mirrored in phenomena traditionally discussed under the heading of 'cue integration') and likewise idiosyncratic principles by which it glues together fragmented structures of partially activated conceptual forms into a globally more coherent structure (as witnessed, e.g. by phenomena pertaining to so-called impossible objects, or the so-called dual nature of picture perception).

Conceptual forms have their own properties, which can be rather surprising when viewed exclusively from the perspective of an adaptive coupling to the external world. Clearly, the organism as an entirety must be adapted to the specific circumstances and biologically relev-

\footnotetext{
${ }^{8}$ I will use the notion of 'data type' in an informal way. Note, however, that a data type together with its associated operations can be regarded as a computational module. In this sense, the availability of conceptual forms themselves can be regarded as an extreme variant of modularity. Also from the perspective of evolutionary biology, abstract data types and modularity are intimately connected. Modularity presumably is, at all levels of biological organisation, the basis for the evolvability of complex systems and a driving force in their evolution (e.g. Kirschner \& Gerhart, 1998; Wagner, Mezey \& Calabretta, 2005). An increase in modularisation with respect to sensory input systems and with respect to the internal systems that take advantage of the sensory information results, however, in an increasing number of interface problems that the system has to solve. Accordingly, we can reasonably speculate that the biological emergence of abstract data formats has its evolutionary origin in the requirement of solving these interface problems (cf. Mausfeld, in press b). More specifically, it emerges from the computational needs to tie together an increasing number of subsystems in a data format that is sufficiently abstract for an integration of the information provided by different subsystems. In this sense, the biological tendency for an increasing amount of modularisation spurs and enforces, with respect to computational systems, an increase in data abstraction.
} 
ant properties of the environment in which it has evolved. From this, however, no substantial constraints - beyond the weakest ones of some structural consistency - can be derived as to the data formats or conceptual forms underlying perceptual computations. These forms can neither be reasonably assumed to mirror 'corresponding' aspects of the external world nor to be 'optimally' adapted to specific features of the physical environment. Our most complex perceptual achievements - for instance seeing dispositional properties of objects (e.g. pertaining to material qualities), intentional properties of objects (e.g. tools), or mental states of others - were, in the evolutionary development of the functional architecture of the perceptual system, only made possible by decoupling the data types of the Perceptual System from the given sensory information and by furnishing the Perceptual System with conceptual forms that go far beyond anything expressible in sensory terms. Theoretical considerations and findings of evolutionary biology (e.g. Webster \& Goodwin, 1996; Gould, 2002) suggest that, just like other biological traits and design features of the organism, the structure of the conceptual forms, on which the computations of the perceptual system are based, cannot simply be derived from alleged adaptive requirements. Rather these conceptual forms will, in their evolutionary development, most likely be essentially co-determined by constraints that stem from the fact that only certain physical and computational channels were open as feasible evolutionary paths (cf. Carroll, 2005). The conceptual forms of the Perceptual System must not only be adequate with respect to the external world (however one understands such a requirement); they must also be computationally adequate, i.e. they have to fit into the entire computational architecture.

With respect to the functional architecture of the brain, the Perceptual System possesses interfaces to various other systems, notably to higher order interpretative systems, where meanings are assigned in terms of 'external world' properties. It generates options for patterns of activated conceptual forms, given a sensory input, which are interpretable by these external systems. At present, we still have only the most rudimentary ideas and theoretical speculations about the higher order systems that take advantage of the outputs provided by the Perceptual System. Much more is known, however, about what is regarded here as the Sensory System and its computational principles. Hence, more specific conjectures about the relation between the Sensory and the Perceptual System can be inferred from the available experimental evidence. I will again attempt to abstractly characterise a few fairly general principles that appear to me well-motivated and empirically well-supported in the light of findings that have been accrued in various fields.

On the present account, the Sensory System and the Perceptual System have their own and proprietary types of data formats. Moreover can the conceptual forms of the Perceptual System, over which its computations are performed, not be derived within the computational apparatus of the Sensory System. Conceptual forms are logically autonomous in the sense that they cannot be achieved by mathematical transformations of the sensory input. The relation between these two systems can therefore only be mediated by some interface function. Such an interface function has to take the output of the Sensory System as an argument and to call a set of conceptual forms. The notion of an interface function thus captures and makes more 
precise the intuitions that are usually expressed by the locutions that a certain "sensory information triggers, or is a cue or sign for" some semantic categorisation. I will therefore refer to such an interface function also as a triggering function. Although not much is presently known about the specific properties of the conceptual forms of the Perceptual System and their triggering functions, certain features can be tentatively derived from computational considerations within the rich constraints provided by perceptual phenomena.

Computational considerations suggest that the codes provided by the Sensory System at its interface with the Perceptual System serve a dual function. Firstly, they activate appropriate conceptual forms and thus determine the potential data formats of the Perceptual System in terms of which input properties are to be exploited. Secondly, they assign concrete values to the free parameters of the activated conceptual forms. Thus, with regards to the Perceptual System, the sensory codes serve as 'instructions' for the activations and specification of conceptual forms. The sensory input opts, as it were, for the activation of a system of conceptual forms with which the system is biologically endowed. This process is constrained by internal requirements of the Perceptual System that pertain to the more global coherence of the activated conceptual forms. Therefore, it is reasonable to regard it as a dynamic bi-directional process geared toward achieving some state of optimal global stability in terms of the internal constraints of the Perceptual System. Presumably, we can account for the "creative forces" (von Szily, 1921, p. 971) of perception, as described and emphasised by Descartes, the Gestaltists, Michotte and many others, already by properties of the Perceptual System. In a sense, conceptual forms can be a regarded as actively seeking the kind of sensory codes (and thus the sensory information) by which they are most completely activated. Structurally, these "creative forces" could have their basis in an underspecification of the conceptual forms with respect to a given input. Underspecification means that the elements that figure in the logical structure of a conceptual form are not fully specified by a given input. Such a semantic underspecification can serve as the structural basis of special computational mechanisms to computationally handle problems of ambiguity and vagueness. The system can achieve a higher degree of flexibility and global stability, if changes, following small variations in the input, in the conceptual forms triggered and in the values of their free parameters are, intuitively speaking, kept at a minimum, particularly at the interfaces of the Perceptual System with subsequent systems. Such a strategy would protect the system from settling, under 'impoverished' situations, on some definite interpretation that would have to be changed to an entirely different interpretation following a small variation in the input. In addition, and independent from issues of the handling of impoverished input situations, underspecified conceptual forms boost the potency of generative processes and enhance the conceptual versatility of the Perceptual System. By routinely operating with underspecified conceptual forms, the Perceptual System can simultaneously provide different layers of semantic 'interpretations', a remarkable capacity that pervades our perceptual and cognitive domains (Mausfeld, in press b).

The theoretical perspective tentatively and abstractly outlined above attempts to condense and make more explicit certain theoretical intuitions that have fruitfully guided systematic investigations of perception since their historic origins, and that have, more recently, been reinvig- 
orated by a wealth of convergent findings from perceptual psychology, ethology, and developmental psychology. Nevertheless, our theoretical understanding of the principles underlying perception still barely scratches the surface. Thus, this theoretical perspective is, needless to say, still very skeletal and in need of precision and specification. However, in comparison with currently prevailing approaches to perception, which are imbued by deeply entrenched common-sense conceptions about perception, much has already been gained, if one takes seriously the besetting foundational question that any successful explanatory account of perception eventually has to answer: What are the abstract internal principles by which a specific biological system, namely the perceptual system, can generate, on the basis of physical spatiotemporal energy patterns, outputs that are organised in terms of semantic categories? The theoretical perspective outlined above enables us to ask novel and promising questions about the principles underlying perception and hence opens up new lines of enquiry. This can be strikingly illustrated by a unique perceptual attribute, namely the attribute of 'realness'. On traditional accounts, this attribute is not considered as providing a serious theoretical challenge for perceptual psychology, because it is usually regarded as a description of the input or the underlying scene. In fact, however, the assignment of the attribute 'realistic' to an input is an achievement of the perceptual system, and thus in need of theoretical explanation. This was emphasised by Metzger (1941) and particularly by Michotte, who conceived of "phenomenal reality" as a "dimension of our visual experience", which he regarded as being closely linked to the internal attribute "potential for being manipulated." (Michotte, 1948/1991, p. 181) On the above account, this global attribute of 'phenomenal reality' seems to be based on special principles of the Perceptual Systems by which it evaluates, in a given input situation, the internal global coherence of a system of activated conceptual forms, the degree to which these forms are specified by the input, and other criteria. Because the criteria for an assignment of the attribute 'real' to an entirety of a situation (which also pertains to the experience of a spatio-temporal continuity as a person) are entirely determined by the internal semantics of the perceptual system, the properties of this attribute and its triggering conditions are unpredictable and surprising from our ordinary perspectives on perception. Instructive examples can be found in the field of computer graphics rendering, where one attempts to construct computer models of perceptual environments or scenes by which images on a screen can be generated that appear realistic, either globally, as in the case of a virtual reality setting, or with respect to certain aspects, such as the material appearances of the depicted objects. These problems of rendering have an interesting counterpart in art history. The simulation of material appearances on a canvass had been regarded as a particular challenge in painting, notably in Dutch renaissance art (cf. Gombrich, 1976). Although already Alberti, in his Trattato della pittura (1435/1972), recognised that by a proper juxtaposition of white and black only, the impressions of gold, silver and glass can be elicited, a realistic impression of material colours in painting turned out to be exceedingly difficult to achieve. 


\section{The perception of material qualities}

Our perceptual system is, evidently, endowed with a rich internal vocabulary, as it were, for 'material qualities'. This vocabulary, which is, on the present theoretical perspective, coded in the conceptual forms of the Perceptual System, pertains to types of material, such as 'skin', 'soil', 'stone', 'metal', 'wood', 'water', and a copiousness of attributes associated with it, such as 'malleable', 'rigid', 'soft', 'taut' (these terms function only as linguistic makeshift descriptions for the internal attributes). The perception of types of material and material qualities, whether visual, haptic or auditory, cannot be understood as simply mirroring corresponding external world properties and hence as being derivable from the sensory input. Of course, as any other perceptual attribute, they have a physical basis in the sense that their triggering conditions are tied to a set (or rather a medley) of external physical regularities. However, the particular perceptual attribute has to be internally available as part of the structure of the type of 'perceptual object' to which it pertains. The attribute itself and its specific structural properties cannot be understood by extensionally describing it by its triggering conditions. This is generally acknowledged for attributes such as 'colour' or, in the auditory domain, attributes referred to as 'tonal volume' or 'sound spaciousness' (cf. Blauert, 1997). Nonetheless, we are usually inclined to believe that perceived material qualities mirror corresponding world properties more than colours do.

Of course, material appearances, as any other perceptual attribute, are tied to structural regularities of the external world. From this, however, nothing follows as to whether the specific classes of regularities that are tied together by the organism to perceptual categories correspond to organism-independent 'natural classes' of physics. The claim that our perceptual system has evolved under rich physical regularities is a truism (in fact, infinitely many physical regularities of any degree of 'oddness' can be formulated). However, there is, trivially, no apriori notion of what constitutes an organism-relevant physical regularity. With respect to perception theory, what can be regarded as a relevant regularity of the world entirely depends on the structure of the organism under scrutiny, such as its size, the spatial and temporal integration properties of receptors and other neural structures, the properties of its memory and its conceptual capacities. Consequently, it is in particular the biologically given conceptual endowment of the perceptual system that determines which regions of the parameter space of the physical world are regarded as 'environment' and hence can function as triggering conditions for perceptual categories. Thus, the kind of image properties by which specific material appearances of 'perceptual objects' can be elicited will most likely depend as much on the structure of the conceptual forms involved as on external world regularities.

Issues pertaining mainly to ecological physics and rendering recently have revived investigations of material appearances. These investigations revealed that material appearances, such as lustre, silk (Koenderink \& Pont, 2002), translucency (e.g. Fleming \& Bülthoff, 2005) or gloss (e.g. Fleming, Dror \& Adelson, 2003; Wendt, Faul \& Mausfeld, 2008), have exceedingly intricate triggering conditions. They can be invoked by a multiplicity of combinations of specific ranges of image parameters. As expected, some subsets of the triggering conditions, 
by which a given material appearance can be elicited, can be related to regularities of the ecological physics of the 'corresponding' type of material, i.e. to regularities in the way light interacts with certain types of physical surfaces. Yet, the entirety of triggering conditions for a certain material appearance, i.e. the equivalence classes of input properties that are tied to a certain material appearance, cannot be derived from or reduced to external physical regularities. These equivalence classes, which extensionally describe the internal semantic categories for material qualities of perceptual objects, are determined by the structure of the conceptual forms of the Perceptual System. Because of this, the triggering conditions for material appearances are given by a rather motley conglomerate of physical conditions, which has about the same degree of 'naturalness' as, e.g., metameric colour classes have with respect to the wavelength composition of lights. Unsurprisingly then, material appearances can also be evoked by input conditions that are physically incorrect or incoherent (e.g. Fleming \& Bülthoff, 2005) with respect to a generation process caused by a 'corresponding' type of material. For the endeavour to gain insights into the structure of the conceptual forms in which material appearances figure as an internal attribute, it is therefore an essential task to identify the complex triggering conditions by which they can be evoked. The more idiosyncratic with respect to external physical regularities the triggering conditions turn out to be, the more likely will they shed some light on the logical form of the 'perceptual objects' to which these attributes, as part of their conceptual form, belong.

In perceptual psychology, investigations of perceptual attributes pertaining to material qualities have been, apart from a few exceptions, almost entirely neglected. This was predominantly due to its theoretical pre-occupation, notably in psychophysics, with elementary attributes (in particular attributes that appear to be directly related to specific elementary coding processes of a single sensory modality and that are not too difficult to be manipulated experimentally). Attributes for material qualities or the type of perceptual object to which they belong, however, are intrinsically transmodal in character and thus go, in particular, far beyond purely visual attributes. Therefore, they defy, more than seemingly elementary attributes, traditional attempts to understand them in terms of sensory input properties and properties inferentially derived from these (unless one tacitly presumes the internal availability of corresponding semantic categories). This was recognised not only by Gestaltists but also by many thinkers outside prevailing lines of thinking. For instance, Schapp (1910) - in his dissertation supervised by Husserl - emphasised: "The things as perceived own a surplus of properties which are not simply coloured surfaces and which cannot be obtained by associations or inferences from other sensory properties. One directly sees tenacity, brittleness, obdurateness, bluntness and many other attributes for which we lack linguistic descriptions." Similar insights were expressed with respect to the complex attributes of perceptual objects that pertain to perceived possibilities for actions, notably by Jakob von Uexkuell, Wolfgang Köhler ("requiredness"), Kurt Koffka ("demand character"), Egon Brunswik ("intentional object"), or James Gibson ("affordances") (Gibson, however, differed from the other ones in that on his conception of affordances 'meanings' are externalised altogether). 
However, two interesting exceptions to this traditional neglect of material appearances can be found in the classic literature, namely investigations, notably by Helmholtz, of stereoscopic lustre, and Katz' enquiries into modes of appearances.

\section{Modes of colour appearance}

The pre-occupation with apparently elementary attributes that can allegedly be investigated more or less in isolation had a particularly detrimental effect on enquiries into colour perception. Traditional colour science has almost exclusively been based on a notion of 'pure' colour or 'colour per se', which can be studied independently from the conceptual structure or internal semantics of the perceptual system and hence independently from the type of 'perceptual object' to which a corresponding attribute belongs. Consequently, perceptual attributes pertaining to material qualities have been regarded not as primordial attributes but rather as derivative ones that can be inferred from certain combinations of colours, luminance textures, etc. In fact, however, empirical as well as theoretical evidence strongly suggests that material qualities are primordial and that the attribute of 'colour per se' is predominantly based on cultural and technology-driven abstractions (cf. Mausfeld, 2003).

The perceptual primacy, as it were, of material colours is also mirrored in the way we linguistically exploit the output of the perceptual system. For instance, Hochegger (1884, p. 36) found it "remarkable that etymological investigations on abstract colour names always find the roots in words that mean shiny, glowing, burning, shimmering, dingy, burnt, etc. Even the expressions for colours which seem to be abstract are, in fact, not primordial but rather emerged from paleness, brightness, glossy, matt, dingy etc." In the transition from the ancient Greek's emphasis on forms of light, such as brightness, lustre, and the changeability of colours to the subsequent culturally-shaped progression toward an increasingly abstract colour vocabulary, we can observe a shift from colour appearances as material properties and 'forms of light' to an abstractive notion of 'colour per se' as an intrinsic object property. (Of course, the fact that our perceptual and cognitive capacities provide us with the means to arrive at an abstract notion of ,colour per se' is of theoretical interest and in need of explanation in itself.)

Katz (1911), following Hering, was clearly aware that 'colour' is intimately interwoven with the organisation of 'space' - in his felicitous terms, a "marriage of colour and space" - and thus cannot be studied in isolation. By his "modes of appearance", Katz provided a descriptive account for the fact that qualities of colour intrinsically depend on the way in which the 'perceptual objects' to which 'colours' are attached are organised in perceptual space.

Accordingly, Katz distinguished various categories of phenomenal appearance, such as 'surface colours', 'volume colours', or 'illumination colours', each of which exhibits distinctive phenomenological characteristics and different coding properties with respect to other perceptual attributes. The guiding idea behind this classification is that the appearances of colour phenomenally segregate into mutually exclusive categories because they mirror, as Gelb $(1929$, p. 600) put it, internal processes or states of "essentially different nature" (whereas on the Standard Model of Perception, the different 'modes of appearance' are simply regarded as 
context-dependent modifications of 'colour per se'). Accordingly, 'colour' does not constitute a unitary or homogeneous attribute; rather, colour-related parameters figure, with different coding properties, in the structure of different types of conceptual forms (cf. Mausfeld, 2003). Katz' classification of modes of colour appearance already captures basic intuitions underlying investigations of material appearances.

\section{Lustrous appearances and their triggering conditions}

The other exception to the traditional neglect of material appearances are investigations of the phenomenon of stereoscopic lustre, which was recognised as of great theoretical importance by Helmholtz, Brücke, Wundt, Kirschmann, Bühler and many others. The phenomenon in point can be easily demonstrated by the stimulus used by Helmholtz (1867) and displayed in figure 8 .

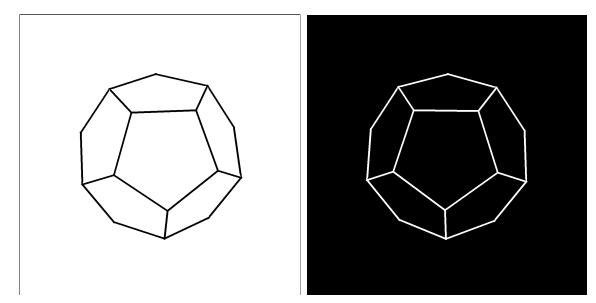

Fig. 8: Helmholtz display for stereoscopic lustre

Under stereoscopic viewing conditions, the binocular combination of the two line drawings of inverted luminance contrast yields a vivid lustrous appearance. Similar appearances can be produced by a variety of different highly reduced stimulus configurations, both under binocular and monocular viewing conditions. It is of particular theoretical interest that lustrous appearances can be elicited by highly impoverished stimulus conditions that do not contain cues pertaining to texture, luminance gradients, or specular highlights. The classic literature comprises a range of studies that attempted to identify critical image parameters for lustrous appearances and that showed that the kind of image properties by which lustre can be elicited is exceedingly variegated and can consist of relatively simple input features. There are also instructive similarities with respect to the auditory perception of material qualities. Corresponding experiments (e.g. Klatzky, Pai \& Krotkov, 2000; Carello, Wagman \& Turvey, 2005) show that also in this domain relatively elementary features act as a triggering basis for assigning complex material properties to the 'auditory objects' involved. Furthermore, material properties of 'auditory objects' are intrinsically transmodal in character as well. This again suggests that the perception of material properties critically depends on structural properties of the conceptual forms involved.

\section{Lustrous appearances and the internal segregation of accidental and essential components}

The attribute of lustre pertains to a property of perceptual objects of the type 'surface' that captures a specific structural relation with respect to another type of conceptual form, namely 'illumination'. This structural relation is, in turn, part of the entire organisation of perceptual space, 
into which 'surfaces' and 'illumination' are embedded. The attribute of lustre apparently denotes an intrinsic property of a special class of 'surfaces', namely those that can make visible, via their spatial relations to an 'illumination', intrinsic properties of the 'illumination' itself as well as certain aspects of the spatial relation between the specific instances of 'surface' and 'illumination'. In the local sensory input, both types of triggering conditions are inextricably entangled. Thus, special computational means are required, to disentangle them. As in the cases of related achievement, such as disentangling colour-related properties of 'surfaces' and 'illumination', the latter functions primarily as a medium to attain intrinsic properties of the former. Thus, the computational task, as it were, that the Perceptual System faces is to perform a causal analysis in terms of its conceptual forms, by which it can disentangle what is regarded, in the internal semantics of the system, as accidental properties of 'surfaces' and what is regarded as essential or intrinsic properties.

The internal causal analysis, which underlies a segregation of intrinsic and accidental aspects of 'surfaces', seems to go along with, or probably is even based on, an internal segregation of both causal components into different spatial layers. This idea is in particular suggested by the phenomenological observations that lustrous appearances, both for monocular and binocular viewing conditions, exhibit some kind of phenomenal segmentations into two different (shallow) depth layers. This observation has been extensively reported and discussed in the classical literature on this topic (e.g. Bixby, 1928). The shallow depth segmentation involved in almost all material colour appearances is again evidence for the "marriage of colour and space" in the internal structural organisation of corresponding attributes.

Hering (1879), who clearly recognised how intimately the attribute of colour is interwoven with the internal organisation of perceptual space, therefore places, in line with his internalist inclinations, lustrous appearances entirely within his discussion of the organisation of perceptual space. According to Hering (1879, p. 576), lustrous appearances arise as a consequence of a shallow depth segmentation with respect to the percept of a surface by which an "essential" and an "accidental" colour component of a surface are disentangled. Such a "cleavage of sensation" into shallow depth layers arises when there is a "surplus of light" with respect to the acceptable values for 'surfaces' and 'ambient illuminations'. The sensory input pattern is, on Hering's account, internally sliced into perceptual layers, which pertain to internal representations of different types, namely to a 'surface' type and to an 'illumination'-type, the specific interrelations of which result in the activation of the kind of 'surface'-type attribute that codes a specific internal 'material quality'. The components represented by the different perceptual layers involved are taken to be related to the functional achievement of causally disentangling, with respect to the sensory input, from illumination properties an intrinsic surface attribute, namely lustre, that cannot simply be captured by descriptions in terms of geometrically homogeneous spectral reflectance distributions of matte surfaces. Although Hering's account of lustrous appearances as part of the organisation of perceptual space remained highly sketchy, it draws attention to important aspects of the conceptual forms involved (including those for global spatial aspects) and their organisation. 


\section{Glassy appearances and the internal organisation of space}

Material appearances, of which Katz' "modes of appearance" are a special case, seem to intrinsically depend on the way in which the 'perceptual objects' to which corresponding attributes are attached are organised in perceptual space. Because of this, the triggering conditions for the attribute 'lustre' and its structural properties can only be understood in intimate connection with the structural and computational organisation of spatial aspects. This becomes even more apparent in the case of another attribute for material appearance, namely 'glassiness'.

In the classic literature, several observations were reported that, for certain stimulus configuration that typically involve static presentations of stereoscopically fused images of 3D-objects or 3D-scenes, a filling of space in-between the objects seems to occur. Karpinska, (1910, p. 44ff.) reported that, under certain conditions, in stereoscopic viewings of line drawings the space in-between the lines appears to be filled with something that has no other material quality than solidity. Schumann (1920) attached to Karpinska's observations a particular importance for a theoretical understanding of the organisation of space. Schumann reported that under his stereoscopic viewing conditions, the empty, interspace' appears as if filled with a certain kind of material that his subjects described as ,vitreous and tangible” (p. 228), reminding them of „glass“, ,ice“, „gelatine“, or „frozen air“ (p. 232). His subjects also reported that, with respect to the discernible depth layer that is nearest to the observer, this glassy appearance looks as if it belonged to a fronto-parallel surface. Subjects, however, differed to the degree to which they see this layer as extending in spatial depth. While some saw the glassy layer as rather thin, others reported it as filling the entire space. Möller (1925) followed up Schumann's investigations and performed an extensive range of observations into this phenomenon, using a great variety of different stimulus conditions. Since then, corresponding phenomena, albeit occasionally reported, have not received systematic theoretical or experimental interest. Unlike lustrous appearances and their triggering conditions, the occurrence of glassy appearances under these viewing conditions does not seem to be easily amenable to interpretations in terms of internal causal analyses (and, to an even lesser extent, to interpretations in terms of external world regularities). Rather these occurrences seem to mirror internal properties of the way attributes are attached to conceptual forms pertaining to surfaces and the organisation of space. Within the theoretical framework outlined here, the occurrence of glassy appearances under highly impoverished input conditions can be understood as the result of a constitutive property of the perceptual system, viz. its capability to computationally operate on semantically underspecified conceptual forms (Mausfeld, in press b). On this conception, glassy appearances occur under input conditions by which conceptual forms for 'surfaces' are activated whose parameters for lightness, colour or texture cannot be assigned values by the sensory input.

The phenomenon of a glassy appearance that fills the entire 'spatial medium' can be illustrated by the stimulus configuration displayed in figure 10 . The stereoscopically combined stimulus consists of a random array of white dots on a grey background. In the first stimulus configuration (upper row), the white dots of the two monocular half-images have different disparities, 
which were randomly chosen for each dot. The dots therefore appear, in the fused image, as lying in different depth planes. In the second stimulus configuration (lower row), the two monocular half-images are identical and thus appear as lying in a fronto-parallel plane. For the images in which the dots appear as occupying different positions in 3D-space, observers in experiments that Gunnar Wendt and I conducted described the situation as showing a 'snow flurry' and reported that the 'snow flakes' are embedded in a block of 'solid glass'. No such material appearance of the 'interspace' was reported for the lower row half-images, in which the dots do not have different disparities. The appearance of the points being embedded in solid glass did not even disappear when each point moved along its own trajectory during the presentation. Rather, observers reported that the snow flakes moved freely through a block of solid glass.

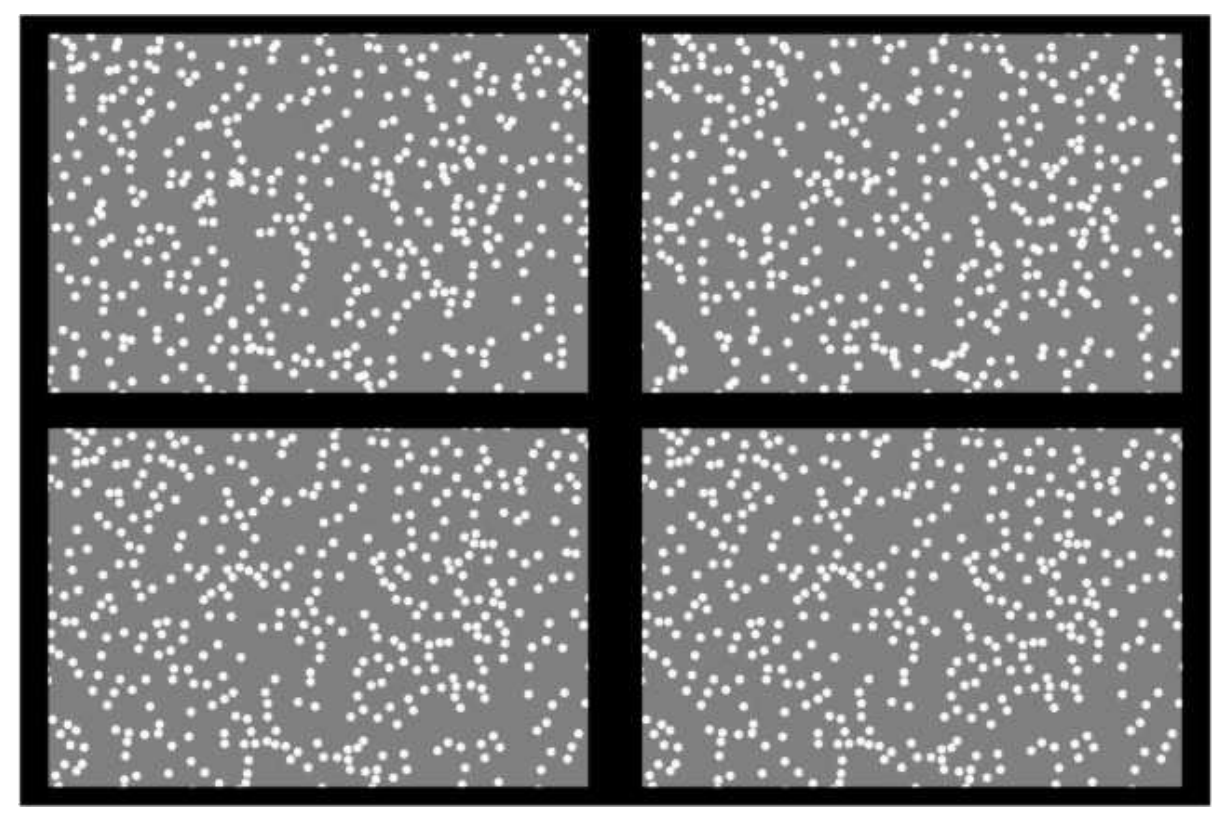

Fig. 10: Two stereo-pairs of ,snow flurry' (upper row: white points have different disparities; lower row: all points have the same stereo disparity)

These and other experiments on the triggering conditions of certain material appearances illustrate that the triggering conditions for material appearances and the structural and computational properties of their internal organisation are surprising and often highly unexpected from a perspective that focuses predominantly on external regularities. However, a system that is as complex and has an evolutionary history as long as our perceptual system is not solely constrained by the adaptational requirement of coupling the organism as an entirety as best as possible to its environment. Its functional architecture, including the kind of data formats or conceptual forms over which its computations are performed, will strongly be co-determined by a great variety of internal constraints, ranging from physical to computational ones, that arise during the evolutionary development of a system of this complexity. The nature of the conceptual forms underlying perception will most likely be formed and shaped by powerful internal constraints, within the apparently rather broad latitude of design options that are left open by global adaptational restrictions. Investigations of phenomenal material qualities seem to be par- 
ticularly rewarding for the endeavour to better understand the structural form of our 'perceptual objects' and, along with that, some of the abstract principles of perception on which the achievements of our perceptual system are based.

\section{Acknowledgments}

I thank Reinhard Niederée for stimulating conversations and Franz Faul for valuable probing comments from a dedicated externalist's perspective. I also thank the editors for helpful suggestions. This work was supported by BMBF-grant 01GWS060 and DFG-grants MA 1025/10-3 and 1025/10-4.

\section{References}

Albertazzi, L. 2006. Immanent realism. Introduction to Brentano. Berlin: Springer.

Baillargeon, R. 1993. The object concept revisited: New directions in the investigation of infant's physical knowledge. In Visual Perception and Cognition in Infancy, ed. C.E. Granrud, 265-315. Hillsdale: New Jersey.

Bonatti, L., Frot, E., Zangl, R. \& Mehler, J. 2002. The human first hypothesis: Identification of conspecifics and individuation of objects in the young infant. Cognitive Psychology, 44: $388-426$.

Bixby, F.L. 1928. A phenomenological study of luster. Journal of General Psychology, 1:136-174.

Blauert, J. 1997. Spatial Hearing: The Psychophysics of Human Sound Localization. Cambridge, Mass.: MIT Press.

Brentano, F. 1874/1995. Psychology from an Empirical Standpoint. London: Routledge.

Brentano, F. 1874/1973. Psychologie vom empirischen Standpunkte. Hamburg: Meiner.

Burke, L. 1952. On the tunnel effect. Quarterly Journal of Experimental Psychology, 4: 121138.

Caramazza, A. \& J. R. Shelton. 1998. Domain-specific knowledge systems in the brain: The animate inanimate distinction. Journal of Cognitive Neuroscience, 10: 1-34.

Carello, C., Wagman, J.B., \& Turvey, M.T. 2005. Acoustic specification of object properties. In Moving image theory: Ecological considerations, ed. J.D.Anderson \& B.F. Anderson, 79104. Carbondale, IL: Southern Illinois University Press.

Carroll, S. B. 2005. Endless Forms Most Beautiful: The New Science of EvoDevo. New York: W. W. Norton \& Company.

Casati, R. 2005. Common-sense, philosophical, and theoretical notions of an object: Some methodological problems. The Monist 88: 571-599.

Chomsky, N. 1966. Cartesian Linguistics. A Chapter in the History of Rationalist Thought. New York: Harper \& Row. 
Chomsky, N. 1996. Power and Prospect. Reflections on Human Nature and the Social Order. London: Pluto Press.

Chomsky, N. 2000. New Horizons in the Study of Language and Mind. Cambridge:

Cambridge University Press.

Descartes, R. 1642/1985. Meditations of First Philosophy. In The Philosophical Writings of Descartes, transl. J. Cottingham, R. Stoothoff \& D. Murdoch,Vol. 2. Cambridge: Cambridge University

Fleming, R.W. \& Bülthoff, H.H. 2005. Low-level image cues in the perception of translucent materials. ACM Transactions on Applied Perception2: 346-382.

Flombaum, J.I. \& Scholl, B.J. 2006. A temporal same-object advantage in the tunnel effect: Facilitated change detection for persisting objects. Journal of Experimental Psychology: Human Perception and Performance 32: 840 - 853.

Fodor, J.A. 1980. Fixation of belief and concept acquisition. In Language and Learning: The Debate between Jean Piaget and Noam Chomsky, ed. M. Piatelli-Palmarini, 142-149.

Cambridge, Mass.: Harvard University Press.

Gallistel, C.R. 1998. Symbolic processes in the brain: the case of insect navigation. In Methods, models and conceptual issues. An invitation to cognitive science, eds. D. Scarborough \& S. Sternberg, Vol. 4, 1-51. Cambridge, Mass.: MIT Press.

Gaukroger, S. 1990. The background to the problem of perceptual cognition. In Arnauld: On True and False Ideas, transl. Gaukroger, S. Manchester: Manchester University Press.

Gelb, A. 1929. Die 'Farbenkonstanz' der Sehdinge. In Handbuch der normalen und pathologischen Physiologie, ed. A. Bethe, G.v. Bergmann, G. Embden, \& A. Ellinger, Bd.12, 1.Hälfte. Receptionsorgane II, 594-678. Berlin: Springer.

Gombrich, E.H. 1976. The Heritage of Apelles. Studies in the Art of the Renaissance. Ithaca: Cornell University Press.

Gould, S.J. 2002. The Structure of Evolutionary Theory, Cambridge, Mass: Harvard University Press.

Grier, M. 2001. Kant's Doctrin of Transcedental Illusion. Cambridge: Cambridge University Press.

Hamlin, J. K., Wynn, K. \& Bloom, P. 2007. Social evaluation by preverbal infants. Nature 450: $557-559$.

Hare, B., Call, J., Agnetta, B. \& Tomasello, M. 2000. Chimpanzees know what conspecifics do and do not see. Animal Behavior 59: 771-785.

Hauser, M.D., Pearson, H.M. \& Seelig, D. 2002. Ontogeny of tool use in cotton-top tamarins Saguinus oedipus: innate recognition of functionally relevant features. Animal Behaviour 64: 299-311.

Heider, F. \& Simmel, M. 1944. An experimental study of apparent behavior. American Journal of Psychology 57: 243-249

Helmholtz, H. von 1856. Ueber die Erklärung der stereoskopischen Erscheinung des Glanzes. Verhandlungen der naturhistorischen Vereinigung der Rheinlande, 28-40.

Helmholtz, H. von 1867. Handbuch der physiologischen Optik. Hamburg: Voss. 
Hering, E. 1879. Der Raumsinn und die Bewegungen des Auges. InHandbuch der Physiologie der Sinnesorgane, ed. L. Hermann, Bd. 3, 343-601. Leipzig: Vogel.

Hinzen, W. 2006. Mind Design and Minimal Syntax. Oxford: Oxford University Press.

Hochegger, R. 1884. Die geschichtliche Entwicklung des Farbensinnes. Innsbruck: Wagner'sche Universitätsbuchhandlung.

Jackendoff, R. 1987. Consciousness and the Computational Mind. Cambridge, Mass.: MIT Press.

Jackendoff, R. 2002. Foundations of language. Oxford: Oxford University Press.

Karpinska, L. v. 1910. Experimentelle Beiträge zur Analyse der Tiefenwahrnehmung. Zeitschrift für Psychologie 57: 1-89.

Katz, D. 1911. Die Erscheinungsweisen der Farben und ihre Beeinflussung durch die Individuelle Erfahrung. Leipzig: Barth.

Kirschner, M., \& Gerhart, J. 1998. Evolvability. Proceedings of the National Academy of Sciences of the United States of America 95: 8420-8427.

Klatzky, R.L., Pai, D.K. \& Krotkov, E.P. 2000. Perception of material from contact sounds, Presence: Teleoperators and Virtual Environments, 9: 399-410.

Koenderink, J. J. \& Pont, S. 2002. The secret of velvety skin. Machine Vision and Applications; Special Issue on Human Modeling, Analysis and Synthesis.

Köhler, W. 1947. Gestalt Psychology. New York: Liveright.

Leslie, A. 1994. ToMM, ToBY, and agency: Core knowledge and domain specificity. In Mapping The Mind: Domain Specificity In Cognition And Culture, ed. L. A. Hirschfeld \& S.A. Gelman, 119-148. Cambridge: Cambridge University Press.

Lorenz, K. 1941/1982. Kant's doctrine of the a priori in the light of contemporary biology. In Learning, development, and culture: Essays in evolutionary epistemology, ed. H. C. Plotkin, 121-143. Chichester: Wiley. (Originally published as Kants Lehre vom apriorischen im Lichte gegenwärtiger Philosophie: Blätter für Deutsche Philosophie, 1941 15: 94-125.)

Ludlow, P. 2003. Referential semantics for I-languages? In Chomsky and His Critics, ed. L.M. Antony \& N. Hornstein , 140-161. Oxford: Blackwell.

Mausfeld, R. 2002. The physicalistic trap in perception. In Perception and the Physical World, eds. D. Heyer \& R. Mausfeld,75-112. Chichester: Wiley.

Mausfeld, R. 2003. 'Colour' as part of the format of two different perceptual primitives: The dual coding of colour. In Colour Perception: Mind and the Physical World, ed. R. Mausfeld \& D. Heyer, 381-429. Oxford: Oxford University Press.

Mausfeld, R. (2010a). Colour within an internalist framework: The role of 'colour' in the structure of the perceptual system. In Color Ontology and Color Science, ed. J. Cohen \& M. Matthen, 381-429. Cambridge, Mass.: MIT Press

Mausfeld, R. (2010 b). Intrinsic multiperspectivity: On the architectural foundations of a distinctive mental capacity. In Cognition and Neuropsychology: International Perspectives on Psychological Science, Vol. 1, ed. Frensch, P. \& R. Schwarzer, 95-116. London: Psychology Press. 
Metzger, W. 1941. Psychologie. Die Entwicklung ihrer Grundannahmen seit Einführung des Experiments. Darmstadt: Steinkopff.

Michotte, A, 1948/1991. L'énigma psychologique de la perspective dans le dessin linéaire. Bulletin de la Classe des Lettres de l'Académie Royale de Belgique 34 : 268-288. (The psychological enigma of perspective in outline pictures, In Michotte's Experimental Phenomenology of Perception, eds. G. Thinès, A. Costall \& G. Butterworth 1991. Hillsdale, NJ: Erlbaum.)

Michotte, A. 1954/1991. Autobiography. In Michotte's Experimental Phenomenology of Perception, ed. G. Thinès, A. Costall \& G. Butterworth 1991. Hillsdale, NJ: Erlbaum.

Möller, E.F. 1925. The "glassy sensation”. American Journal of Psychology 36: 249-285.

Moran, D. 1996. Brentano's thesis. Proceedings of the Aristotelian Society, Supplementary Volume 70: 1-27.

Neisser, U. 1976 Cognition and reality: Principles and implications of cognitive psychology. New York: W.H. Freeman

Premack, D. \& Premack, A.J. 1995. Intention as Psychological Cause. In Causal Cognition, eds. D. Sperber, D. Premack \& A.J. Premack, 185-199. Oxford: Clarendon.

Premack, D, \& Premack, A. 2003. Original Intelligence. Unlocking the Mystery of Who We Are. New York: McGraw-Hill.

Pustejovsky, J. 1995. The Generative Lexicon. Cambridge, Mass.: MIT Press.

Santos, L.R., Hauser, M.D., Spelke, E.S. 2001. Recognition and categorization of biologically significant objects by rhesus monkeys (Macaca mulatta): the domain of food. Cognition 82: 127-155.

Schapp, W. 1910. Beiträge zur Phänomenologie der Wahrnehmung. Göttingen.

Scholl, B. J., \& Tremoulet, P. 2000. Perceptual causality and animacy. Trends in Cognitive Sciences 4: 299 - 309.

Scholl, B. J. 2007. Object persistence in philosophy and psychology. Mind \& Language 22: $563-591$.

Schumann, F. 1920. Die Repräsentation des leeren Raumes im Bewußtsein. Eine neue Empfindung. Zeitschrift für Psychologie, 85, 224-244.

Shimojo, S. \& Shams, L. 2001. Sensory modalities are not separate modalities: plasticity and interactions. Current Opinion in Neurobiology 11: 505-509.

Smith, B. 1994. Austrian Philosophy. The Legacy of Franz Brentano. LaSalle, Il. \& Chicago: Open Court.

Spelke, E.S. 1990. Principles of object perception. Cognitive Science 14: 29-56.

Spelke, E.S. 2000. Core knowledge. American Psychologist 55: 1233-1243.

Spelke, E.S., Gutheil, G., \& van der Walle, G. 1995. The development of object perception. In Visual Cognition. An Invitation to Cognitive Science, eds. S.M. Kosslyn \& D. Osherson, Vol. 2, 297-330. Cambridge, Mass.: MIT Press.

Strawson, G. 2003. Real materialism. In Chomsky and his Critics, eds. L. Antony \& N. Hornstein. 49-88. Oxford: Blackwell. 
Tinbergen, N. 1963. On aims and methods of ethology. Zeitschrift für Tierpsychologie 20: 410-433

Trevarthen, C. 1998. The concept and foundations of infant intersubjectivity. In Intersubjective Communication and Emotion in Early Ontogeny, ed. Bråten, 15-46. Cambridge: Cambridge University Press.

von Szily, A. 1921. Stereoskopische Versuche mit Schattenrissen. Gräfes Archiv für Ophtalmologie, 105: 964-972.

Wagner, G.P., Mezey, J. \& Calabretta, R. 2005. Natural selection and the origin of modules. InModularity. Understanding the Development and Evolution of Complex Natural Systems, ed. W. Callabaut \& D. Rasskin-Gutman, 33-49. Cambridge, Mass.: MIT Press.

Wang, R.F. \& Spelke, E.S. 2002. Human spatial representation: Insights from animals, Trends in Cognitive Sciences 6: 376-382.

Webster, G. \& Goodwin, B.C. 1996. Form and Transformation. Generative and Relational Principles in Biology. Cambridge: Cambridge University Press.

Wehner, R. 2003. Desert ant navigation: how miniature brains solve complex tasks. Journal of Comparative Physiology, A 189: 579-588.

Wendt, G., Faul, F. \& Mausfeld, R. 2008. Highlight disparity contributes to the authenticity and strength of perceived glossiness. Journal of Vision 8: 1-10.

Yang, C.D. 2002. Knowledge and Learning in Natural Language. Oxford: Oxford University Press.

Yolton, J.W. 1984. Perceptual Acquaintance from Descartes to Reid. Minneapolis: University of Minnesota Press.

Yolton, J.W. 1996. Perception and Reality. A History from Descartes to Kant. Ithaca: Cornell University Press. 